\title{
Article \\ Synergistic Effect of Polyphenol-Rich Complex of Plant and Green Propolis Extracts with Antibiotics against Respiratory Infections Causing Bacteria
}

\author{
Anna Ramata-Stunda ${ }^{1, * \mathbb{D}}$, Zaiga Petriṇa ${ }^{2}$, Valda Valkovska ${ }^{3}$, Mārtiṇs Borodušḳis ${ }^{1}$, Līga Gibnere ${ }^{4}$, \\ Eleonora Gurkovska ${ }^{4}$ and Vizma Nikolajeva ${ }^{2, *}$ \\ 1 Department of Microbiology and Biotechnology, Faculty of Biology, University of Latvia, 1 Jelgavas Str., \\ LV-1004 Riga, Latvia; martins.boroduskis@lu.lv \\ 2 Microbial Strain Collection of Latvia, University of Latvia, 1 Jelgavas Str., LV-1004 Riga, Latvia; \\ zaiga.petrina@lu.lv \\ 3 Faculty of Chemistry, University of Latvia, 1 Jelgavas Str., LV-1004 Riga, Latvia; valda.valkovska@lu.lv \\ 4 Silvanols Ltd., 2 Kurbada Str., LV-1009 Riga, Latvia; liga.gibnere@silvanols.lv (L.G.); \\ eleonora.gurkovska@silvanols.lv (E.G.) \\ * Correspondence: anna.ramata-stunda@lu.lv (A.R.-S.); vizma.nikolajeva@lu.lv (V.N.)
}

check for updates

Citation: Ramata-Stunda, A.; Petrina, Z.; Valkovska, V.; Boroduškis, M.; Gibnere, L.; Gurkovska, E.;

Nikolajeva, V. Synergistic Effect of Polyphenol-Rich Complex of Plant and Green Propolis Extracts with Antibiotics against Respiratory Infections Causing Bacteria. Antibiotics 2022, 11, 160. https:// doi.org/10.3390/antibiotics11020160

Academic Editors: Tomás González Villa, Trinidad de Miguel and Marta Martins

Received: 30 November 2021

Accepted: 20 January 2022

Published: 26 January 2022

Publisher's Note: MDPI stays neutral with regard to jurisdictional claims in published maps and institutional affiliations.

Copyright: (c) 2022 by the authors. Licensee MDPI, Basel, Switzerland. This article is an open access article distributed under the terms and conditions of the Creative Commons Attribution (CC BY) license (https:// creativecommons.org/licenses/by/ $4.0 /)$.

\begin{abstract}
Bacterial infections are a prevalent complication after primary viral respiratory infections and are associated with high morbidity and mortality. Antibiotics are widely used against bacterial respiratory pathogens; however, the rise in antibiotic-resistant strains urges us to search for new antimicrobial compounds, including ones that act synergistically with antibiotics. In this study, the minimal inhibitory (MIC) and minimal bactericidal (MBC) concentrations of a polyphenolrich complex of green propolis, Tabebuia avellanedae bark, and Olea europaea leaf extracts against Staphylococcus aureus, Haemophilus influenzae, and Klebsiella pneumoniae were determined, followed by an analysis of the synergistic effect with clarithromycin, azithromycin, and amoxiclav (875/125 mg amoxicillin/clavulanic acid). A combination of extracts showed activity against all three bacterial strains, with MIC values ranging from 0.78 to $12.5 \mathrm{mg} / \mathrm{mL}$ and $\mathrm{MBC}$ values from 1.56 to $12.5 \mathrm{mg} / \mathrm{mL}$. The extracts showed synergistic activity with azithromycin and clarithromycin against S. aureus, with clarithromycin against K. pneumoniae, and with all three tested antibiotics against $H$. influenzae. Synergy with clarithromycin was additionally evaluated in a time-kill assay where the synergistic effects against $S$. aureus and K. pneumoniae were seen within the first $6 \mathrm{~h}$ of incubation. The results show the potential of polyphenol-rich extracts in enhancing the efficacy of antibiotic therapy and indicate their potential to be used in the management of respiratory infections.
\end{abstract}

Keywords: polyphenols; synergy; respiratory infections; green propolis; Olea europea leaf extract; Tabebuia avellanedae bark

\section{Introduction}

Respiratory tract infections are extremely prevalent, being among the most diagnosed diseases in primary and secondary care. Bacterial infections, especially pneumonia, are a common complication after primary infection with respiratory viruses such as influenza viruses, rhinoviruses, and coronaviruses and are often characterized by severe disease and high mortality. Common bacterial pathogens associated with respiratory tract infections are Haemophilus influenzae, Streptococcus pneumoniae, Branhamella (Moraxella) catarrhalis, Staphylococcus aureus, Streptococcus pyogenes, Klebsiella pneumoniae, and Pseudomonas aeruginosa. Clinical challenges of these infections associated with increased rates of antimicrobial resistance are observed among these pathogens [1-3].

Antibiotics are widely used in the treatment of respiratory tract infections. The preferred treatment for bacterial infections is generally broad-spectrum antibiotics, but this can result in undesirable side effects that have a negative impact on the normal host 
microflora. To avoid disease progression, complications, and negative outcomes, alternative approaches to eliminate bacterial pathogens are necessary [1,2]. Although different groups of antibiotics with different mechanisms of action are available, increasing rates of antibiotic resistance limit their use and efficacy. Inappropriate prescription and use of antibiotics, along with a lack of new effective antimicrobials, make the situation even worse [4,5]. To confront multidrug-resistant pathogens as well as the emergence of new strains, a reevaluation of the choice and need for antibiotics, and the search for new antimicrobial compounds are needed [6,7].

Plants are valuable sources of antimicrobial compounds. There are over 1340 plants with known antimicrobial activity, and over 30,000 antimicrobial plant-derived compounds have been characterized [8]. Current studies and applications include both purified forms of specific plant metabolites as well as crude extracts. Unlike antibiotics, bacteria rarely develop resistance to plant products [9]. Another advantage is the lack of adverse effects characteristic of conventional antibiotics [10-12]. Particularly interesting are phenolic compounds, one of the most diverse groups of secondary plant metabolites with various biological functions, including antibacterial, antiviral, antifungal, antioxidative, and anti-inflammatory activities. Phenolic compounds act on the bacterial cell membrane, interfere with nucleic acid synthesis, inhibit bacterial metabolism, coagulate cytoplasmic proteins, and interfere with biofilm formation. Apart from direct antimicrobial activity, plant secondary metabolites have indirect activities, such as stimulation of the host's immune response and modification of resistance mechanisms. The activity of extracts or isolated compounds varies due to the chemical composition and structures of the compounds $[8,13,14]$. Several plant-derived antimicrobials are synergistic with antibiotics; thus, they can be combined with standard antibiotic therapy for enhanced efficacy. The potential of plant compounds to substitute antibiotics in the case of resistant strains has attracted interest and has been investigated [13,15-20].

GoImmune Strong ${ }^{\circledR}$ is a registered food supplement with antioxidative activity and is recommended for use in the cold season to promote immunity. The product contains a standardized mixture of Brazilian green propolis extract, olive (Olea europea) leaf extract, and tabebuia (Tabebuia avellanedae) bark extract. The composition was developed based on data in scientific literature. After the launch, follow-up of product use pointed to the potential efficacy for prevention of respiratory infections and strengthening immunity during an illness. These observations raised a practical question about the concomitant use of this herbal product and different antibiotics in case of bacterial infection.

O.europea is well known for its antibacterial and antifungal activity, and substances, including oleuropein, oleanolic acid, hydroxytyrosol, and tyrosol, have been identified as the compounds that provide this activity. Different parts of the olive are used; however, leaves have been identified as the best source of antimicrobial substances. Although purified olive leaf compounds have shown noteworthy activity, it has been reported that crude extracts are more effective, indicating the synergistic activities among the individual constituents of O. europaea extracts [21-26]. Reports on the spectrum of olive leaf antimicrobial activity are contradictory. In some studies, activity against a few microorganisms was detected [22,26], while others report broad activity against both Gram-negative and Gram-positive bacteria, including respiratory pathogens S. aureus and K. pneumoniae [27]. The synergy between antibiotics and olive leaf extracts has been reported. The combination of ampicillin with polyphenol-rich olive leaf extract or individual biophenols resulted in a better antibacterial effect against $S$. aureus and Escherichia coli than ampicillin alone [28].

Propolis, another component of the GoImmune Strong ${ }^{\circledR}$ complex, is a resinous substance that honeybees (Apis mellifera) and stingless bees (Melipona mondury, M. scutellaris) produce by mixing their salivary gland excretions with exudate from the buds, leaves, stems, branches, and bark of plants. The chemical composition of propolis depends on its geographical origin, local flora, the species of bee, and the season [29]. The antimicrobial effects of propolis against various bacteria, yeasts, and viruses are well documented. These properties have been attributed to phenolic compounds [30-38]. Propolis extracts exert their 
antibacterial potential using two distinct mechanisms: either by promoting host immune responses or via direct interaction with bacterial cells. Efficacy against Gram-positive bacteria dominates, with Gram-negative bacteria being less susceptible. Activity against S. aureus is most frequently reported [39-42]. The potent bacteriostatic and bactericidal effects of propolis can be explained by its combined action on the inhibition of protein synthesis, bacterial growth, and cell lysis [42]. A combination of propolis and antibiotics to enhance the efficacy of antibacterial therapy appears promising. Propolis exerts synergistic effects with antibiotics acting on the bacterial wall synthesis and ribosome function, but it does not seem to interact with antibiotics acting on nucleic acids or folic acid biosynthesis [42-48]. Synergy with antibiotics against respiratory pathogens has been shown [49,50]. Different types of propolis are used in the food and pharmaceutical industries. Brazilian green propolis is derived from apical buds and young leaves of Baccharis dracunculifolia. This is one of the most studied types of propolis, with antibacterial activity against Gram-positive bacteria reported as a characteristic feature [50-53].

Plants from the genus Tabebuia are used in traditional medicine in Latin American countries and are known for their anti-inflammatory, antimicrobial, and anticancer activities. Tabebuia avellanedae bark extracts inhibit the growth of Gram-positive bacteria. The antimicrobial effect is attributed to the phenolic compound lapachol and other naphthoquinones that are the main components of Tabebuia spp. extracts. Limited data are available about the mechanism of antimicrobial activity and its interaction with other antimicrobial substances. It is supposed that phenolic compounds of Tabebuia induce oxidative stress in the bacterial cell membrane and interfere with ATP synthesis [54-57].

The accumulated evidence from various published studies on the antibacterial activity of individual components of the GoImmune Strong ${ }^{\circledR}$ complex and the growing need for improved strategies to tackle bacterial infections encouraged us to investigate the in vitro efficacy of the complex against respiratory pathogens and to evaluate its synergy with selected antibiotics, with the aim to repurpose the complex for prophylaxis and therapy of respiratory infections.

\section{Results}

\subsection{Total Content of Polyphenols}

The total phenolic content of individual extracts as well as the GoImmune Strong ${ }^{\circledR}$ complex was determined using Folin-Ciocalteu assay (Table 1). O.europaea extract had the highest concentration of total polyphenols; however, a high content was detected in the other two extracts as well. The polyphenol concentration in the combination of the extracts was $63.37 \mathrm{mg} / \mathrm{g}$ DW.

Table 1. Total phenolic content of individual extracts and extract complex, gallic acid equivalents mg/g DW. TAE-Tabebuia avellanedae bark extract; OEE-Olea europaea leaf extract; GPE-green propolis extract. Mean \pm SD from three independent analyses.

\begin{tabular}{cccc}
\hline TAE & OEE & GPE & GoImmune Strong $^{\circledR}$ \\
\hline $23.56 \pm 3.37$ & $171.69 \pm 27.50$ & $13.03 \pm 2.12$ & $63.37 \pm 10.89$ \\
\hline
\end{tabular}

\subsection{Chemical Composition}

A chromatographic analysis confirmed the presence of the characteristic compounds in all three extracts. A total of 37 compounds, including 10 unidentified, were found in olive leaf extract (Table 2.). Oleuropein, hydroxytyrosol, and verbascoside were the dominating compounds. A total of 48 compounds were identified in Tabebuia avellanedae bark extract, with hydroxybenzoic acid, verbascoside, isoverbascoside, with derivatives of rutin and quercitin being the dominating ones (Table 3). Chromatographic analysis of green propolis extract revealed the high concentrations and diversity of flavonoids, a total of 51 compounds were found. Among dominating compounds, p-coumarinic acid, coumaric acid prenylesters, dicaffeoylquinic acid isomers, and 4-hydroxy-3-prenylcinnamic 
acid were identified (Table 4). Results show that all three extracts are rich in phenolic compounds, including those that have been characterized before for their antimicrobial activity. The content of oeleuropein was lower than that claimed by the manufacturer of the olive leaf extract, and analysis did not confirm the presence of lapachol in tabebuia bark extract. These differences might be due to the variations in sample preparation and analytical methods used.

Table 2. Compounds identified in the Olea europaea leaf extract.

\begin{tabular}{|c|c|c|c|c|c|}
\hline Compound & Formula & $(\mathbf{M}-\mathrm{H})^{-}$ & $\mathrm{RT}$, min & Refs. & $\begin{array}{c}\text { Quantity } \\
\text { Mean } \pm \text { SD, } \mu \mathrm{g} / \mathrm{g} \text { DW }\end{array}$ \\
\hline Hydroxytyrosol ${ }^{b, c}$ & $\mathrm{C}_{8} \mathrm{H}_{10} \mathrm{O}_{3}$ & 153.0557 & 7.12 & {$[27,58]$} & $1695 \pm 11^{\mathrm{d}}$ \\
\hline Hydroxytyrosol glucoside $\mathrm{b,c}$ & $\mathrm{C}_{14} \mathrm{H}_{20} \mathrm{O}_{8}$ & 315.1085 & 7.78 & [59] & $203 \pm 8^{d}$ \\
\hline Oleoside $b, c$ & $\mathrm{C}_{16} \mathrm{H}_{22} \mathrm{O}_{11}$ & 389.1089 & 7.94 & [60] & $44 \pm 6^{\mathrm{k}}$ \\
\hline 4-Hydroxybenzoic acid ${ }^{a}$ & $\mathrm{C}_{7} \mathrm{H}_{6} \mathrm{O}_{3}$ & 137.0244 & 8.35 & {$[61]$} & $41 \pm 3$ \\
\hline Aesculin b,c & $\mathrm{C}_{15} \mathrm{H}_{16} \mathrm{O}_{9}$ & 339.0722 & 8.59 & {$[60]$} & $21.8 \pm 0.9^{\mathrm{d}}$ \\
\hline Chlorogenic acid $^{\text {a }}$ & $\mathrm{C}_{16} \mathrm{H}_{18} \mathrm{O}_{9}$ & 353.0878 & 9.01 & [59] & $17 \pm 2$ \\
\hline $\mathrm{NI}^{\mathrm{c}}$ & - & 377.1487 & 9.39 & - & NQ \\
\hline Luteolin rutinoside $b, c$ & $\mathrm{C}_{27} \mathrm{H}_{30} \mathrm{O}_{16}$ & 609.1461 & 9.61 & {$[62]$} & $5.7 \pm 0.5^{j}$ \\
\hline C10 isoprenoid ${ }^{\mathrm{C}}$ & $\mathrm{C}_{17} \mathrm{H}_{24} \mathrm{O}_{11}$ & 403.1246 & 9.67 & [63] & NQ \\
\hline $\mathrm{NI}^{\mathrm{c}}$ & - & 305.0739 & 9.95 & & $\mathrm{NQ}$ \\
\hline Demethyloleuropein b,c & $\mathrm{C}_{24} \mathrm{H}_{30} \mathrm{O}_{13}$ & 525.1614 & 10.05 & [63] & $4.0 \pm 0.2^{\mathrm{k}}$ \\
\hline Hydroxyphenylacetic acid b,c & $\mathrm{C}_{8} \mathrm{H}_{8} \mathrm{O}_{3}$ & 151.0401 & 10.09 & [64] & $0.858 \pm 0.004^{d}$ \\
\hline Rutin ${ }^{a}$ & $\mathrm{C}_{27} \mathrm{H}_{30} \mathrm{O}_{16}$ & 609.1461 & 10.18 & {$[21,27,58]$} & $7.5 \pm 0.2$ \\
\hline Verbascoside $b, c$ & $\mathrm{C}_{29} \mathrm{H}_{36} \mathrm{O}_{15}$ & 623.1981 & 10.22 & {$[21,27]$} & $120.4 \pm 0.6^{\mathrm{e}}$ \\
\hline p-Coumaric acid ${ }^{a}$ & $\mathrm{C}_{9} \mathrm{H}_{8} \mathrm{O}_{3}$ & 163.0401 & 10.26 & {$[58,61]$} & $1.80 \pm 0.04$ \\
\hline Luteolin O-glucoside $^{b, c}$ & $\mathrm{C}_{21} \mathrm{H}_{20} \mathrm{O}_{11}$ & 447.0933 & 10.41 & {$[27,58,60]$} & $31.0 \pm 0.6^{j}$ \\
\hline Isoverbascoside $\mathrm{b}, \mathrm{c}$ & $\mathrm{C}_{29} \mathrm{H}_{36} \mathrm{O}_{15}$ & 623.1981 & 10.49 & [27] & $18.8 \pm 1.3^{\mathrm{e}}$ \\
\hline Ferulic acid ${ }^{a}$ & $\mathrm{C}_{10} \mathrm{H}_{10} \mathrm{O}_{4}$ & 193.0506 & 10.60 & {$[58,61]$} & $1.6 \pm 0.2$ \\
\hline Apigenin 7-O-glucoside ${ }^{b, c}$ & $\mathrm{C}_{21} \mathrm{H}_{20} \mathrm{O}_{10}$ & 431.0984 & 10.88 & {$[21,27]$} & $4.6 \pm 0.4^{\mathrm{j}}$ \\
\hline Luteolin O-glucoside b,c & $\mathrm{C}_{21} \mathrm{H}_{20} \mathrm{O}_{11}$ & 447.0933 & 10.93 & {$[21,27,60]$} & $17.3 \pm 1.1^{j}$ \\
\hline Elenolic acid ${ }^{b, c}$ & $\mathrm{C}_{11} \mathrm{H}_{14} \mathrm{O}_{6}$ & 241.0718 & 11.03 & [59] & $7.2 \pm 0.4^{\mathrm{k}}$ \\
\hline Oleuropein $^{a}$ & $\mathrm{C}_{25} \mathrm{H}_{32} \mathrm{O}_{13}$ & 539.1770 & 11.10 & {$[27,58-60]$} & $8681 \pm 85$ \\
\hline $\mathrm{NI}^{\mathrm{c}}$ & - & 601.2146 & 11.61 & - & NQ \\
\hline Elenolic acid derivative $b, c$ & - & 241.0718 & 12.16 & - & $2.5 \pm 0.3^{\mathrm{k}}$ \\
\hline Luteolin $b, c$ & $\mathrm{C}_{15} \mathrm{H}_{10} \mathrm{O}_{6}$ & 285.0405 & 12.20 & {$[58,60]$} & $18 \pm 2^{j}$ \\
\hline Quercetin $b, c$ & $\mathrm{C}_{15} \mathrm{H}_{10} \mathrm{O}_{7}$ & 301.0354 & 12.22 & [58] & $21 \pm 2^{j}$ \\
\hline $\mathrm{NI}^{\mathrm{c}}$ & - & 377.1253 & 12.47 & - & NQ \\
\hline Apigenin derivative ${ }^{b, c}$ & - & 269.0455 & 12.96 & - & $1.1 \pm 0.2^{j}$ \\
\hline $\mathrm{NI}^{\mathrm{c}}$ & - & 377.1255 & 13.23 & - & $208 \pm 12^{d}$ \\
\hline $\mathrm{NI}^{\mathrm{c}}$ & - & 377.1255 & 13.45 & - & $53 \pm 7^{d}$ \\
\hline Chrysoeriol $^{b, c}$ & $\mathrm{C}_{16} \mathrm{H}_{12} \mathrm{O}_{6}$ & 299.0561 & 14.89 & {$[65]$} & $4.5 \pm 0.2^{\mathrm{j}}$ \\
\hline $\mathrm{NI}^{\mathrm{c}}$ & - & 545.1911 & 14.94 & - & NQ \\
\hline $\mathrm{NI}^{\mathrm{c}}$ & - & 721.3611 & 15.80 & - & NQ \\
\hline Apigenin derivative ${ }^{b, c}$ & - & 269.0455 & 16.02 & - & $1.8 \pm 0.3^{j}$ \\
\hline C30 isoprenoid ${ }^{\mathrm{c}}$ & $\mathrm{C}_{30} \mathrm{H}_{48} \mathrm{O}_{4}$ & 471.3480 & 17.64 & [63] & NQ \\
\hline $\mathrm{NI}^{\mathrm{c}}$ & - & 401.3017 & 20.46 & - & NQ \\
\hline $\mathrm{NI}^{\mathrm{c}}$ & - & 401.3014 & 20.99 & - & NQ \\
\hline
\end{tabular}

NI—not identified; NQ— not quantified; ${ }^{a}$ confirmed by standard; ${ }^{b}$ confirmed by reference; ${ }^{c}$ confirmed by HRMS; ${ }^{\mathrm{d}}$ expressed as p-coumaric acid; ${ }^{\mathrm{e}}$ expressed as caffeic acid; ${ }^{\mathrm{j}}$ expressed as rutin; ${ }^{\mathrm{k}}$ expressed as oleuropein. 
Table 3. Compounds identified in the Tabebuia avellanedae bark extract.

\begin{tabular}{|c|c|c|c|c|c|}
\hline Compound & Formula & $(\mathrm{M}-\mathrm{H})^{-}$ & $\begin{array}{l}\text { RT, } \\
\text { min }\end{array}$ & Refs. & $\begin{array}{c}\text { Quantity } \\
\text { Mean } \pm \mathrm{SD}, \mu \mathrm{g} / \mathrm{g} \mathrm{DW}\end{array}$ \\
\hline Protocatechuic acid derivative ${ }^{c}$ & - & $153.0193 *$ & 6.46 & - & $52 \pm 7^{1}$ \\
\hline Protocatechuic acid ${ }^{a}$ & $\mathrm{C}_{7} \mathrm{H}_{6} \mathrm{O}_{4}$ & 153.0193 & 7.37 & - & $87 \pm 9$ \\
\hline $\begin{array}{l}\text { 2,4-dimethoxyphenyl } \\
\text { 1-O- } \beta \text {-D-apiofuranosyl-(1-6)- } \beta \text {-D-glucopyranoside } \\
\text { b,c }\end{array}$ & $\mathrm{C}_{19} \mathrm{H}_{28} \mathrm{O}_{12}$ & 447.1509 & 7.95 & [66] & $5.61 \pm 0.06^{d}$ \\
\hline 4-Hydroxybenzoic acid ${ }^{\text {a }}$ & $\mathrm{C}_{7} \mathrm{H}_{6} \mathrm{O}_{3}$ & 137.0244 & 8.35 & {$[54,57]$} & $20.5 \pm 0.5$ \\
\hline Epiaucubin $b, c$ & $\mathrm{C}_{15} \mathrm{H}_{22} \mathrm{O}_{9}$ & 345.1191 & 8.41 & [66] & $3.0 \pm 0.4^{\mathrm{k}}$ \\
\hline $\mathrm{NI}^{\mathrm{c}}$ & - & 461.1284 & 8.49 & - & $121 \pm 9^{d}$ \\
\hline $\mathrm{NI}^{\mathrm{c}}$ & - & 487.1431 & 8.55 & & NQ \\
\hline C10 isoprenoids ${ }^{c}$ & $\mathrm{C}_{19} \mathrm{H}_{28} \mathrm{O}_{11}$ & 431.1559 & 8.67 & [63] & NQ \\
\hline Hydroxy benzoic acid isomer $b, c$ & $\mathrm{C}_{7} \mathrm{H}_{6} \mathrm{O}_{3}$ & 137.0244 & 8.70 & [54] & $521 \pm 46^{\mathrm{m}}$ \\
\hline Protocatechuic acid derivative $^{c}$ & - & $153.0193 *$ & 8.73 & - & $9.4 \pm 0.3^{1}$ \\
\hline $\mathrm{NI}^{\mathrm{c}}$ & - & 523.1660 & 9.00 & - & NQ \\
\hline Chlorogenic acid ${ }^{a}$ & $\mathrm{C}_{16} \mathrm{H}_{18} \mathrm{O}_{9}$ & 353.0878 & 9.05 & [54] & $0.49 \pm 0.02$ \\
\hline Protocatechuic acid derivative ${ }^{c}$ & - & $153.0193 *$ & 9.13 & - & $9.4 \pm 0.3^{1}$ \\
\hline Specioside ${ }^{b, c}$ & $\mathrm{C}_{24} \mathrm{H}_{28} \mathrm{O}_{12}$ & 507.1508 & 9.16 & [67] & $2.6 \pm 0.2^{k}$ \\
\hline 10-O-(4-methoxybenzoyl)-impetiginoside $\mathrm{A}$ b,c & $\mathrm{C}_{23} \mathrm{H}_{28} \mathrm{O}_{12}$ & 495.1508 & 9.37 & [66] & $3.1 \pm 0.3^{\mathrm{k}}$ \\
\hline Caffeic acid ${ }^{\mathrm{a}}$ & $\mathrm{C}_{9} \mathrm{H}_{8} \mathrm{O}_{4}$ & 179.0350 & 9.39 & {$[54]$} & $6.87 \pm 0.04$ \\
\hline Flavone or flavonol ${ }^{c}$ & $\mathrm{C}_{29} \mathrm{H}_{28} \mathrm{O}_{11}$ & 551.1559 & 9.41 & {$[63]$} & $30 \pm 4^{j}$ \\
\hline $\mathrm{NI}^{\mathrm{c}}$ & - & 639.1926 & 9.61 & - & NQ \\
\hline $\mathrm{NI}^{\mathrm{c}}$ & - & 521.1521 & 9.74 & - & $62 \pm 6^{\mathrm{d}}$ \\
\hline Calyxin $^{\mathrm{c}}$ & $\mathrm{C}_{35} \mathrm{H}_{34} \mathrm{O}_{8}$ & 581.2181 & 9.83 & [68] & $14.6 \pm 0.4^{\mathrm{i}}$ \\
\hline C15 isoprenoids ${ }^{c}$ & $\mathrm{C}_{15} \mathrm{H}_{16} \mathrm{O}_{6}$ & 291.0874 & 10.11 & [68] & NQ \\
\hline Verbascoside $^{b, c}$ & $\mathrm{C}_{29} \mathrm{H}_{36} \mathrm{O}_{15}$ & 623.1976 & 10.22 & [54] & $355 \pm 14^{\mathrm{e}}$ \\
\hline p-coumaric acid ${ }^{a}$ & $\mathrm{C}_{9} \mathrm{H}_{8} \mathrm{O}_{3}$ & 163.0401 & 10.26 & {$[54]$} & $2.20 \pm 0.04$ \\
\hline $\begin{array}{l}\text { 1-benzyl-[6-p-hydroxybenzoyl]-b-D-glucopyranosyl-(1-3)-b-D- } \\
\text { glucopyranoside b,c or 2-(4-hydroxyphenyl)ethy1, } \\
\text { 1-O-ß3-D-[5-O-(4-hydroxybenzoyl)]-apiofuranosyl-(1-6)- } \beta \text {-D- } \\
\text { glucopyranoside }{ }^{\text {b,c }}\end{array}$ & $\mathrm{C}_{26} \mathrm{H}_{32} \mathrm{O}_{13}$ & 551.1770 & 10.40 & {$[66,69]$} & $26.0 \pm 1.1^{d}$ \\
\hline $\begin{array}{l}\text { 3,4-dimethoxyphenyl 1-O- } \beta \text {-D-[5-O-(4- } \\
\text { methoxybenzoyl)]-apiofuranosyl-(1-6)- } \beta \text {-D-glucopyranoside } \\
\text { b,c }\end{array}$ & $\mathrm{C}_{27} \mathrm{H}_{34} \mathrm{O}_{14}$ & 581.1875 & 10.43 & [66] & $2.11 \pm 0.09^{\mathrm{d}}$ \\
\hline Astragalin $b, c$ & $\mathrm{C}_{21} \mathrm{H}_{20} \mathrm{O}_{11}$ & 447.0933 & 10.46 & [69] & $15.1 \pm 0.8^{\mathrm{j}}$ \\
\hline 5,7-Dihydroxy-3', $4^{\prime}$-dimethoxyflavanone 7-rutinoside ${ }^{c}$ & $\mathrm{C}_{29} \mathrm{H}_{36} \mathrm{O}_{15}$ & 623.1981 & 10.50 & [63] & $265 \pm 20^{i}$ \\
\hline Isoverbascoside ${ }^{b, c}$ & $\mathrm{C}_{29} \mathrm{H}_{36} \mathrm{O}_{15}$ & 623.1976 & 10.53 & [54] & $279 \pm 3^{\mathrm{e}}$ \\
\hline 6-O-(4-methoxybenzoyl)-5,7-bisdeoxycynanchoside b,c & $\mathrm{C}_{23} \mathrm{H}_{30} \mathrm{O}_{12}$ & 497.1664 & 10.56 & [66] & $5.8 \pm 0.3^{\mathrm{k}}$ \\
\hline Veratic acid $b, c$ & $\mathrm{C}_{9} \mathrm{H}_{10} \mathrm{O}_{4}$ & 181.0506 & 10.64 & {$[54,57]$} & $68 \pm 7^{\mathrm{n}}$ \\
\hline C15 isoprenoid ${ }^{\mathrm{c}}$ & $\mathrm{C}_{15} \mathrm{H}_{16} \mathrm{O}_{6}$ & 291.0857 & 10.74 & - & NQ \\
\hline Phenolic compound glycoside $b, c$ & $\mathrm{C}_{27} \mathrm{H}_{34} \mathrm{O}_{15}$ & 597.1825 & 10.81 & [70] & $24 \pm 3$ \\
\hline Veratic acid derivative $^{c}$ & - & 181.0506 * & 11.02 & - & $15 \pm 3^{n}$ \\
\hline $\begin{array}{l}\text { 2-(4-hydroxyphenyl)ethyl, } \\
\text { 1-O- } \beta \text {-D-[5-O-(3,4-dimethoxybenzoyl)]- } \\
\text { apiofuranosyl-(1-6)- } \beta \text {-D-glucopyranoside }{ }^{b, c}\end{array}$ & $\mathrm{C}_{28} \mathrm{H}_{36} \mathrm{O}_{14}$ & 595.2031 & 11.04 & [66] & $70.3 \pm 1.2^{d}$ \\
\hline $\mathrm{NI}^{\mathrm{c}}$ & - & 539.1779 & 11.15 & - & $62 \pm 2^{d}$ \\
\hline $\begin{array}{c}\text { Quercetin 5,7,3',4'-tetramethyl ether Quercetin } \\
5,7,3^{\prime}, 4^{\prime} \text {-tetramethyl ether 3-rutinoside }{ }^{c}\end{array}$ & $\mathrm{C}_{31} \mathrm{H}_{38} \mathrm{O}_{16}$ & 665.2087 & 11.27 & [63] & $230 \pm 19^{j}$ \\
\hline $\begin{array}{l}\text { 2-(4-hydroxyphenyl)ethyl 1-O- } \beta \text {-D-[5-O-(4 methoxybenzoyl)]- } \\
\text { apiofuranosyl-(1-6)- } \beta \text {-D-glucopyranoside }\end{array}$ & $\mathrm{C}_{27} \mathrm{H}_{34} \mathrm{O}_{13}$ & 565.1926 & 11.49 & [66] & $11.8 \pm 1.5^{\mathrm{d}}$ \\
\hline $\mathrm{NI}^{\mathrm{c}}$ & - & 527.1768 & 11.55 & - & $74.1 \pm 1.4^{\mathrm{d}}$ \\
\hline Ferulic acid derivative $^{c}$ & - & $193.0506 *$ & 11.92 & - & $112 \pm 15^{\mathrm{h}}$ \\
\hline Kaempferol deivative ${ }^{c}$ & - & $285.0405 *$ & 12.24 & - & $3.0 \pm 0.3^{j}$ \\
\hline $\mathrm{NI}^{\mathrm{c}}$ & - & 327.2181 & 12.70 & - & NQ \\
\hline $\mathrm{NI}^{\mathrm{c}}$ & - & 329.2345 & 13.14 & - & NQ \\
\hline $\begin{array}{l}\text { 5-hydroxy-2-(1-hydroxyethyl)naphtho [2,3-b]furan-4,9-dione or } \\
\text { 8-hydroxy-2-(1-hydroxyethyl)naphtho [2,3-b]furan-4,9-dione }{ }^{\text {b,c }}\end{array}$ & $\mathrm{C}_{14} \mathrm{H}_{10} \mathrm{O}_{5}$ & 257.0455 & 13.37 & {$[71,72]$} & NQ \\
\hline Alkyl hydroquinone or derivative ${ }^{c}$ & $\mathrm{C}_{17} \mathrm{H}_{26} \mathrm{O}_{4}$ & 293.1758 & 14.72 & [63] & NQ \\
\hline $\mathrm{NI}^{\mathrm{c}}$ & - & 385.2556 & 16.19 & - & NQ \\
\hline $\mathrm{NI}^{\mathrm{c}}$ & - & 285.2042 & 16.42 & - & NQ \\
\hline $\mathrm{NI}^{\mathrm{c}}$ & - & 311.2196 & 17.02 & - & NQ \\
\hline $\mathrm{NI}^{\mathrm{c}}$ & - & 327.1776 & 17.14 & - & NQ \\
\hline
\end{tabular}

NI—not identified; NQ—not quantified; ${ }^{*}-\mathrm{MS}$ fragments; ${ }^{\mathrm{a}}$ confirmed by standard; ${ }^{\mathrm{b}}$ confirmed by reference ${ }^{c}$ confirmed by HRMS; ${ }^{d}$ expressed as p-coumaric acid; ${ }^{\mathrm{e}}$ expressed as caffeic acid; ${ }^{\mathrm{h}}$ expressed as ferulic acid; ${ }^{\mathrm{i}}$ expressed as naringenin; ${ }^{j}$ expressed as rutin; ${ }^{k}$ expressed as oleuropein; ${ }^{1}$ expressed as protocatechuic acid; $\mathrm{m}$ expressed as $\mathrm{p}$-hydroxy benzoic acid; ${ }^{\mathrm{n}}$ expressed as vanillic acid. 
Table 4. Compounds identified in the green propolis extract.

\begin{tabular}{|c|c|c|c|c|c|}
\hline Compound & Formula & $(\mathrm{M}-\mathrm{H})^{-}$ & $\mathrm{RT}$, min & Refs. & $\begin{array}{c}\text { Quantity } \\
\text { Mean } \pm S D, \mu g / g \text { DW }\end{array}$ \\
\hline Chlorogenic acid isomer ${ }^{b, c}$ & $\mathrm{C}_{16} \mathrm{H}_{18} \mathrm{O}_{9}$ & 353.0878 & 8.25 & [73-75] & $15 \pm 2^{f}$ \\
\hline Chlorogenic acid ${ }^{\mathrm{a}}$ & $\mathrm{C}_{16} \mathrm{H}_{18} \mathrm{O}_{9}$ & 353.0878 & 9.05 & {$[50,74]$} & $105 \pm 4$ \\
\hline Caffeic acid ${ }^{a}$ & $\mathrm{C}_{9} \mathrm{H}_{8} \mathrm{O}_{4}$ & 179.0350 & 9.39 & {$[73,76]$} & $45 \pm 6$ \\
\hline Flavone or flavonol ${ }^{\mathrm{c}}$ & $\mathrm{C}_{25} \mathrm{H}_{28} \mathrm{O}_{12}$ & 519.1508 & 9.97 & {$[63]$} & $650 \pm 33^{d}$ \\
\hline p-coumaric acid ${ }^{a}$ & $\mathrm{C}_{9} \mathrm{H}_{8} \mathrm{O}_{3}$ & 163.0401 & 10.26 & {$[73,74,76]$} & $256 \pm 23$ \\
\hline Ferulic acid $^{a}$ & $\mathrm{C}_{10} \mathrm{H}_{10} \mathrm{O}_{4}$ & 193.0506 & 10.52 & {$[73,76]$} & $8.1 \pm 0.6$ \\
\hline Dicaffeoylquinic acid isomer $b, c$ & $\mathrm{C}_{25} \mathrm{H}_{24} \mathrm{O}_{12}$ & 515.1195 & 10.66 & {$[50,73-75]$} & $97 \pm 9^{\mathrm{e}}$ \\
\hline Dicaffeoylquinic acid isomer ${ }^{b, c}$ & $\mathrm{C}_{25} \mathrm{H}_{24} \mathrm{O}_{12}$ & 515.1195 & 10.81 & {$[50,73-75]$} & $97 \pm 6^{\mathrm{e}}$ \\
\hline Dicaffeoylquinic acid isomer $b, c$ & $\mathrm{C}_{25} \mathrm{H}_{24} \mathrm{O}_{12}$ & 515.1195 & 11.00 & {$[50,73-75]$} & $321 \pm 27^{\mathrm{e}}$ \\
\hline Caffeic acid derivative ${ }^{b, c}$ & - & 487.1563 & 11.39 & [73] & $32.9 \pm 0.8^{\mathrm{e}}$ \\
\hline $\begin{array}{l}\text { Luteolin methyl ether crotonylglucoside or } \\
\text { luteolin glucoside methyl butanoate }{ }^{c}\end{array}$ & $\mathrm{C}_{26} \mathrm{H}_{26} \mathrm{O}_{12}$ & 529.1315 & 11.61 & [63] & $17.1 \pm 1.2^{\mathrm{j}}$ \\
\hline Caffeic acid prenyl ester $b, c$ & $\mathrm{C}_{14} \mathrm{H}_{16} \mathrm{O}_{4}$ & 247.0976 & 11.83 & [76] & $18.4 \pm 1.3^{\mathrm{e}}$ \\
\hline Tricaffeoylquinic acid ${ }^{b, c}$ & $\mathrm{C}_{34} \mathrm{H}_{30} \mathrm{O}_{15}$ & 677.1512 & 12.02 & [73] & $98 \pm 9^{\mathrm{e}}$ \\
\hline Dimethyl-dicaffeoylquinic acid ${ }^{b, c}$ & $\mathrm{C}_{27} \mathrm{H}_{28} \mathrm{O}_{12}$ & 543.1508 & 12.13 & [73] & $40 \pm 4^{\mathrm{e}}$ \\
\hline Dimethyl-dicaffeoylquinic acid b,c & $\mathrm{C}_{27} \mathrm{H}_{28} \mathrm{O}_{12}$ & 543.1508 & 12.36 & [73] & $46 \pm 6^{\mathrm{e}}$ \\
\hline 3,4-Dimethyl caffeic acid & $\mathrm{C}_{11} \mathrm{H}_{12} \mathrm{O}_{4}$ & 207.0663 & 12.43 & [76] & $13.6 \pm 1.0^{\mathrm{e}}$ \\
\hline Chlorogenic acid derivative ${ }^{c}$ & - & 353.0878 * & 12.55 & - & $4.2 \pm 0.2^{\mathrm{f}}$ \\
\hline Chlorogenic acid derivative $^{c}$ & - & $353.0878 *$ & 12.71 & - & $3.3 \pm 0.2^{f}$ \\
\hline Naringenin ${ }^{a}$ & $\mathrm{C}_{15} \mathrm{H}_{12} \mathrm{O}_{5}$ & 271.0612 & 12.96 & [73] & $27 \pm 2$ \\
\hline Hesperetin $b, c$ & $\mathrm{C}_{16} \mathrm{H}_{14} \mathrm{O}_{6}$ & 301.0718 & 13.04 & [73] & $270 \pm 16^{\mathrm{i}}$ \\
\hline Kaempferol ${ }^{b, c}$ & $\mathrm{C}_{15} \mathrm{H}_{10} \mathrm{O}_{6}$ & 285.0405 & 13.10 & {$[73,76]$} & $17 \pm 2^{\mathrm{j}}$ \\
\hline Isorhamnetin $b, c$ & $\mathrm{C}_{16} \mathrm{H}_{12} \mathrm{O}_{7}$ & 315.0510 & 13.14 & {$[50,73,76]$} & $21 \pm 3^{j}$ \\
\hline Caffeic acid derivative ${ }^{b, c}$ & - & 705.1835 & 13.31 & [73] & $16 \pm 2^{\mathrm{e}}$ \\
\hline $\mathrm{NI}^{\mathrm{b}, \mathrm{c}}$ & - & 301.1495 & 13.47 & [73] & $25 \pm 2^{d}$ \\
\hline Rhamnetin ${ }^{b, c}$ & $\mathrm{C}_{16} \mathrm{H}_{12} \mathrm{O}_{7}$ & 315.0510 & 13.56 & {$[50,73]$} & $25 \pm 3^{j}$ \\
\hline 3,4-Dihydroxy 5-prenylcinnamic acid ${ }^{b, c}$ & $\mathrm{C}_{14} \mathrm{H}_{16} \mathrm{O}_{4}$ & 247.0976 & 13.61 & {$[50,73-75]$} & $121 \pm 8^{g}$ \\
\hline $\mathrm{NI}^{\mathrm{b}, \mathrm{c}}$ & - & 331.1613 & 13.72 & [73] & $140 \pm 6^{\mathrm{d}}$ \\
\hline Coumaric acid prenyl ester $b, c$ & $\mathrm{C}_{14} \mathrm{H}_{16} \mathrm{O}_{3}$ & 231.1027 & 13.99 & {$[73,76]$} & $301 \pm 11^{\mathrm{d}}$ \\
\hline 4-Hydroxy-3-prenylcinnamic acid b,c & $\mathrm{C}_{14} \mathrm{H}_{16} \mathrm{O}_{3}$ & 231.1027 & 13.99 & {$[50,73-75]$} & $327 \pm 24 \mathrm{~g}$ \\
\hline Caffeic acid benzyl ester $b, c$ & $\mathrm{C}_{16} \mathrm{H}_{14} \mathrm{O}_{4}$ & 269.0819 & 14.45 & {$[73,76]$} & $3.0 \pm 0.3^{\mathrm{e}}$ \\
\hline Coumaric acid derivative $b, c$ & - & 315.1601 & 14.50 & [73] & $56 \pm 7^{\mathrm{d}}$ \\
\hline Kaempferide derivative ${ }^{b, c}$ & - & 377.1957 & 14.60 & [73] & $36.5 \pm 1.3^{j}$ \\
\hline Sakuranetin $\mathrm{b}, \mathrm{c}$ & $\mathrm{C}_{16} \mathrm{H}_{14} \mathrm{O}_{5}$ & 285.0768 & 14.63 & [73] & $23.3 \pm 1.5^{\mathrm{i}}$ \\
\hline Chrysin $b, c$ & $\mathrm{C}_{15} \mathrm{H}_{10} \mathrm{O}_{4}$ & 253.0506 & 14.65 & {$[50,76]$} & $10.1 \pm 0.9^{j}$ \\
\hline Pinocembrin $b, c$ & $\mathrm{C}_{15} \mathrm{H}_{12} \mathrm{O}_{4}$ & 255.0663 & 14.68 & {$[50,73,76]$} & $10.1 \pm 0.7^{\mathrm{i}}$ \\
\hline Kaempferide derivative $b, c$ & - & 377.1953 & 14.75 & [73] & $59 \pm 3^{j}$ \\
\hline Galangin $b, c$ & $\mathrm{C}_{15} \mathrm{H}_{10} \mathrm{O}_{5}$ & 269.0455 & 14.80 & {$[50,76]$} & $7.3 \pm 0.5^{j}$ \\
\hline Caffeic acid phenethyl ester $b, c$ & $\mathrm{C}_{17} \mathrm{H}_{16} \mathrm{O}_{4}$ & 283.0976 & 14.83 & {$[50,73,76]$} & $7.4 \pm 0.2^{\mathrm{e}}$ \\
\hline Kaempferide $b, c$ & $\mathrm{C}_{16} \mathrm{H}_{12} \mathrm{O}_{6}$ & 299.0561 & 14.89 & {$[50,73,76]$} & $110 \pm 11^{\mathrm{j}}$ \\
\hline Dimethoxyquercetin $b, c$ & $\mathrm{C}_{17} \mathrm{H}_{14} \mathrm{O}_{7}$ & 329.0667 & 15.01 & [50] & $33.6 \pm 0.5^{j}$ \\
\hline Dicoumaric prenyl ester $b, c$ & $\mathrm{C}_{23} \mathrm{H}_{22} \mathrm{O}_{6}$ & 393.1344 & 15.08 & {$[73,76]$} & $50 \pm 6^{\mathrm{d}}$ \\
\hline Caffeic acid cinnamyl ester b,c & $\mathrm{C}_{18} \mathrm{H}_{16} \mathrm{O}_{4}$ & 295.0976 & 15.35 & {$[76]$} & $6.5 \pm 0.2^{\mathrm{d}}$ \\
\hline Kaempferide derivative ${ }^{b, c}$ & - & 529.1497 & 15.38 & [73] & $22.4 \pm 0.2^{j}$ \\
\hline Coumaric acid derivative ${ }^{b, c}$ & - & 315.1600 & 15.44 & [73] & $52 \pm 5^{\mathrm{d}}$ \\
\hline Baccharin $^{b, c}$ & $\mathrm{C}_{29} \mathrm{H}_{38} \mathrm{O}_{11}$ & 561.2341 & 15.61 & {$[50,74]$} & $1.5 \pm 0.2^{\mathrm{d}}$ \\
\hline Artepillin $C$ derivative ${ }^{b, c}$ & - & 329.1780 & 15.72 & {$[73]$} & $156 \pm 24^{d}$ \\
\hline Coumaric acid derivative ${ }^{b, c}$ & - & 559.1628 & 15.78 & [73] & $1.81 \pm 0.02^{\mathrm{d}}$ \\
\hline Artepillin $C^{b, c}$ & $\mathrm{C}_{19} \mathrm{H}_{24} \mathrm{O}_{3}$ & 299.1653 & 16.39 & {$[50,73-75]$} & $414 \pm 26^{\mathrm{d}}$ \\
\hline $\mathrm{NI}^{\mathrm{c}}$ & - & 613.2155 & 16.72 & - & NQ \\
\hline $\mathrm{NI}^{\mathrm{c}}$ & - & 613.3219 & 17.50 & - & NQ \\
\hline $\mathrm{NI}^{\mathrm{c}}$ & - & 627.2288 & 17.66 & - & NQ \\
\hline C20 isoprenoid ${ }^{\mathrm{c}}$ & $\mathrm{C}_{20} \mathrm{H}_{30} \mathrm{O}_{2}$ & 301.2226 & 19.14 & [63] & NQ \\
\hline
\end{tabular}

NI-not identified; NQ—not quantified; *-MS fragments; ${ }^{\mathrm{a}}$ confirmed by standard; ${ }^{\mathrm{b}}$ confirmed by reference; ${ }^{c}$ confirmed by HRMS; ${ }^{d}$ expressed as p-coumaric acid; ${ }^{\mathrm{e}}$ expressed as caffeic acid; ${ }^{\mathrm{f}}$ expressed as chlorogenic acid; g expressed as cinnamic acid; ${ }^{i}$ expressed as naringenin; ${ }^{j}$ expressed as rutin.

\subsection{Antibacterial Activity}

Antimicrobial activity assay revealed the activity of the complex against $S$. aureus, $H$. influenzae, and K. pneumoniae (Table 5). The complex was active against all three bacterial strains with MIC ranging from $0.78 \mathrm{mg} / \mathrm{mL}$ in the case of $S$. aureus to $12.5 \mathrm{mg} / \mathrm{mL}$ in the 
case of K. pneumoniae. MBC values ranged from $1.56 \mathrm{mg} / \mathrm{mL}$ for $S$. aureus to $12.5 \mathrm{mg} / \mathrm{mL}$ for H. influenzae and K. pneumoniae.

Table 5. Minimal inhibitory (MIC) and minimal bactericidal (MBC) concentrations GoImmune Strong ${ }^{\circledR}$ complex and selected antibiotics.

\begin{tabular}{|c|c|c|c|c|c|c|}
\hline & \multicolumn{2}{|c|}{ S. aureus } & \multicolumn{2}{|c|}{ K. pneumoniae } & \multicolumn{2}{|c|}{ H. influenzae } \\
\hline & MIC & MBC & MIC & MBC & MIC & MBC \\
\hline Azithromycin $(\mu \mathrm{g} / \mathrm{mL})$ & 0.5 & $>128$ & 8 & $>128$ & 0.25 & $>128$ \\
\hline Clarithromycin $(\mu \mathrm{g} / \mathrm{mL})$ & 0.25 & 128 & 64 & $>128$ & 0.5 & $>128$ \\
\hline Amoxiclav $(\mu \mathrm{g} / \mathrm{mL})$ & 0.13 & 1.0 & 8.0 & 64 & 8 & $>128$ \\
\hline GoImmune Strong $^{\circledR}(\mathrm{mg} / \mathrm{mL})$ & 0.78 & 1.56 & 12.5 & 12.5 & 3.13 & 12.5 \\
\hline
\end{tabular}

\subsection{Combined Effect of GoImmune Strong ${ }^{\circledR}$ Complex and Antibiotics}

\subsubsection{Determination of Fractional Inhibitory Concentrations (FIC) and FIC Index (FICI)}

Checkerboard dilutions were used to test the interaction of GoImmune Strong ${ }^{\circledR}$ antibiotic combinations. Fractional inhibitory concentrations and FIC indexes were calculated to evaluate the synergy of the complex with azithromycin, clarithromycin, and amoxiclav. Three different methods were used to interpret the results (Table 6). Synergy with all tested antibiotics against $H$. influenzae was detected. The complex was synergic against $S$. aureus with azithromycin and clarithromycin, whereas the combination with clarithromycin had the strongest inhibitory effect. For K. pneumoniae, only synergy with clarithromycin, according to Fratini et al.'s 2017 interpretation method, was detected.

Table 6. Fractional inhibitory concentrations (FIC), FIC indexes, and interpretations of them. $\mathrm{FIC}_{\text {antib. }}$-fractional inhibitory concentration of antibiotic; FIC $_{\text {Comp. }}$-fractional inhibitory concentration of the GoImmune Strong ${ }^{\circledR}$ complex.

\begin{tabular}{|c|c|c|c|c|}
\hline Antibio & & Azithromycin & Clarithromycin & Amoxiclav \\
\hline \multirow{4}{*}{ Staphylococcus aureus } & FIC $_{\text {antib. }}$ & 0.25 & 0.06 & 0.5 \\
\hline & FIC $_{\text {Comp }}$ & 0.06 & 0.03 & 0.5 \\
\hline & \multirow[b]{2}{*}{ FICI } & 0.31 & 0.09 & 1.0 \\
\hline & & Synergy ${ }^{a, b, c}$ & Synergy a,b,c & $\begin{array}{c}\text { Additive }^{a}, \\
\text { commutative }^{c} ; \\
\text { no interaction }^{b}\end{array}$ \\
\hline \multirow{4}{*}{ Klebsiella pneumoniae } & FIC $_{\text {antib. }}$ & 1.0 & 0.5 & 0.5 \\
\hline & FIC $_{\text {Comp }}$ & 0.5 & 0.03 & 0.5 \\
\hline & \multirow[b]{2}{*}{ FICI } & 1.5 & 0.53 & 1.0 \\
\hline & & No interaction $b, c$ & $\begin{array}{c}\text { Additive }^{\mathrm{a}} \\
\text { no interaction } \\
\text { synergy }^{\mathrm{c}}\end{array}$ & $\begin{array}{c}\text { Additive }^{\mathrm{a}}, \\
\text { commutative }^{\mathrm{c}} \text {; } \\
\text { no interaction }^{\mathrm{b}}\end{array}$ \\
\hline \multirow{4}{*}{ Haemophilus influenzae } & FIC $_{\text {antib. }}$ & 0.12 & 0.25 & 0.13 \\
\hline & FIC $_{\text {Comp }}$ & 0.25 & 0.13 & 0.25 \\
\hline & \multirow{2}{*}{ FICI } & 0.37 & 0.38 & 0.38 \\
\hline & & Synergy $a, b, c$ & Synergy $a, b, c$ & Synergy ${ }^{a, b, c}$ \\
\hline
\end{tabular}

Methods used for interpretation: ${ }^{a}$ EUCAST, 2000 [77]; ${ }^{\mathrm{b}}$ Odds, 2003 [78]; ${ }^{\mathrm{c}}$ Fratini et al., 2017 [79].

\subsubsection{Time-Kill Assay}

A time-kill kinetic assay was executed using clarithromycin as the antibiotic that had synergy against all three bacteria in combination with the GoImmune Strong ${ }^{\circledR}$ complex. Gram-positive S. aureus as the most susceptible and Gram-negative K. pneumoniae as the 
least susceptible bacteria were chosen for the test. The results of the time kill-kinetic assay of GoImmune Strong ${ }^{\circledR}$ and antibiotic combinations confirmed the results obtained from checkerboard assays (Figures 1 and 2).

GoImmune Strong ${ }^{\circledR}$ alone at 2 MIC and 4 MIC concentrations reduced K. pneumoniae colony counts by $1.52 \log _{10}$ and $2.45 \log _{10}$ after $6 \mathrm{~h}$. The reduction was statistically significant compared to control already after $3 \mathrm{~h}$ incubation $(p<0.001)$. No further reduction was observed over $24 \mathrm{~h}$ period. Clarithromycin did not reduce colony counts. Combination of GoImmune Strong ${ }^{\circledR}$ and clarithromycin reduced colony counts already at 0.5 MIC concentrations within the first $6 \mathrm{~h}$. Strong reduction by $1.6 \log _{10}$ and $3.84 \log _{10}$ was seen after $6 \mathrm{~h}$ incubation in presence of $2 \mathrm{MIC}$ and $4 \mathrm{MIC}$ concentrations. Reduction was statistically significant $(p<0.001)$ compared to control. When compared to clarithromycin alone, after $3 \mathrm{~h}$ incubation, colony count reduction in presence of the combination at $4 \mathrm{MIC}$ concentrations was statistically significant $(p<0.001)$. After $6 \mathrm{~h}$, the reduction in presence of antibiotic and GoImmune Strong ${ }^{\circledR}$ combination was statistically significant compared to clarithromycin alone at all concentrations. Viable bacterial cells were not observed in presence of all concentrations of combination after $24 \mathrm{~h}$ period.

A K.pneumoniae MSCL 535
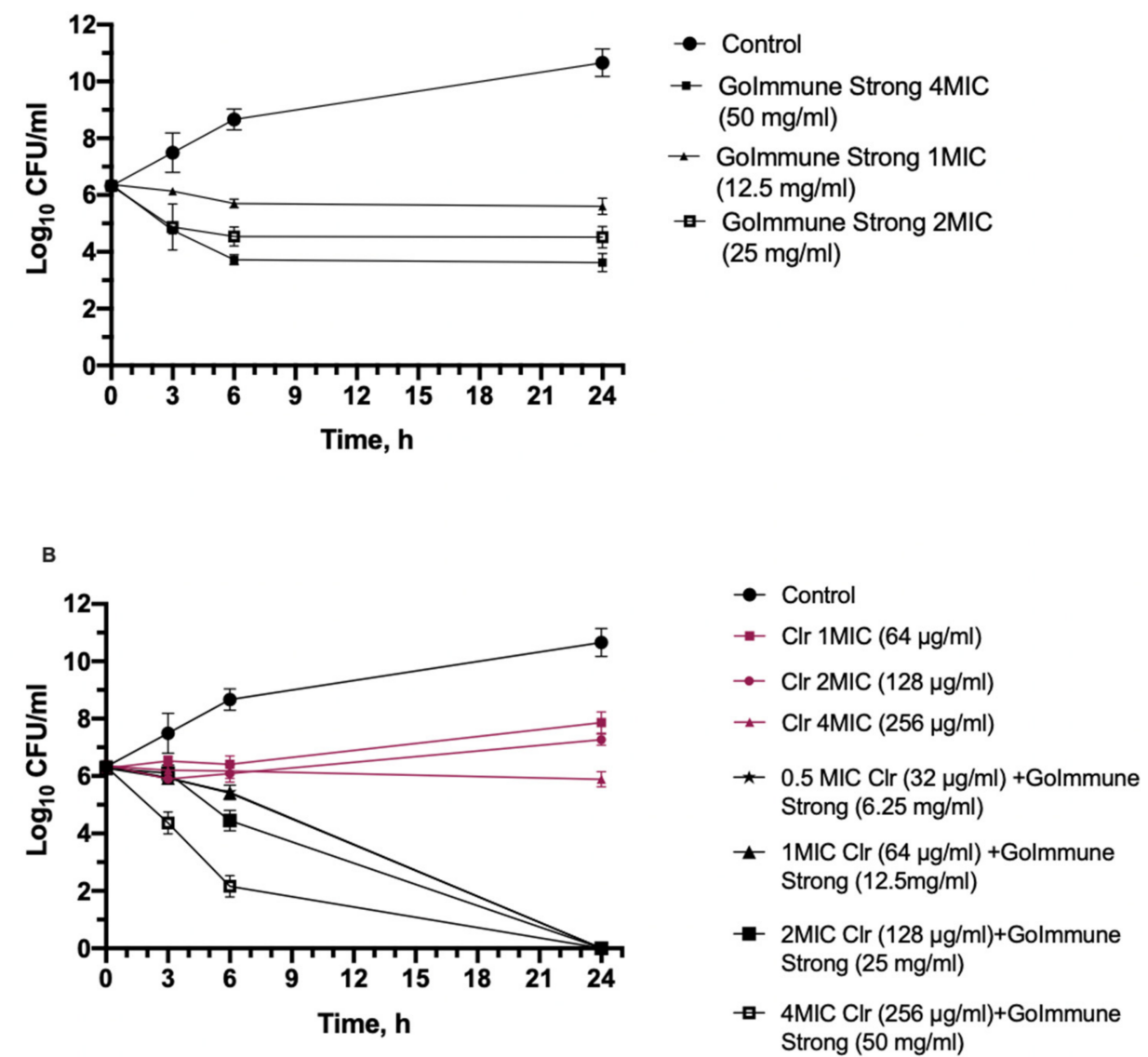

Figure 1. Time-kill curves show synergistic interaction between GoImmune Strong ${ }^{\circledR}$ complex and clarithromycin (Clr) against K. pneumoniae. (A) - time-kill curves of GoImmune Strong ${ }^{\circledR}$ complex at different concentrations. (B)-Time-kill curves of combination of GoImmune Strong ${ }^{\circledR}$ complex and clarithromycin. Each time-curve experiment was performed in duplicate and diluted samples were plated in duplicate on agar plates. Error bars indicate standard deviations. 
S.aureus MSCL 334

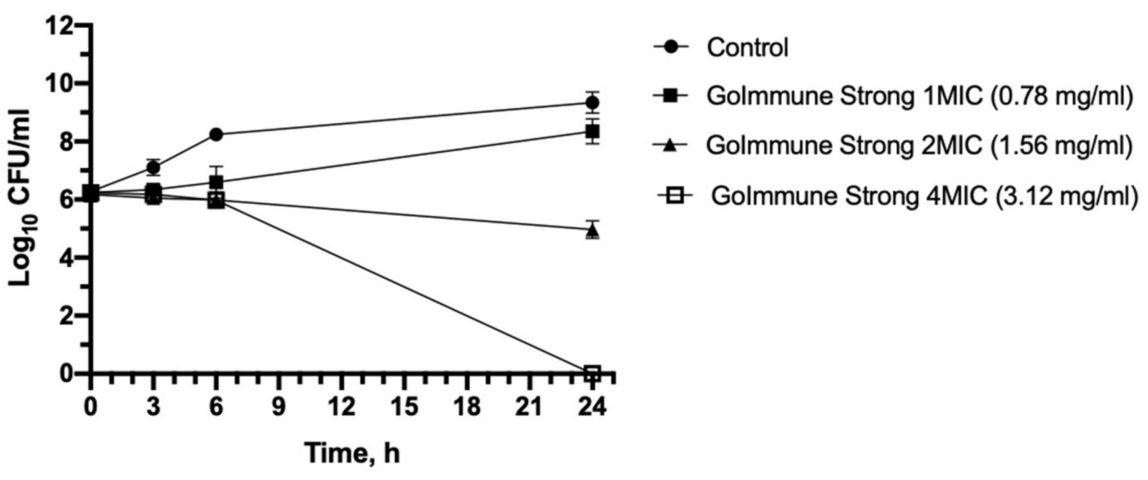

B

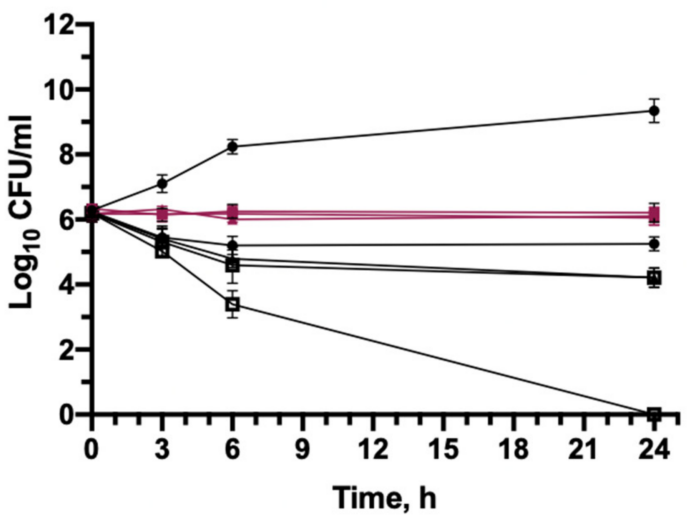

$\rightarrow$ Control

- - $\operatorname{Clr} 1 \mathrm{MIC}(0.25 \mu \mathrm{g} / \mathrm{ml})$

^ $\operatorname{Clr} 2 \mathrm{MIC}(0.5 \mu \mathrm{g} / \mathrm{ml})$

$\rightarrow-\operatorname{Clr} 4 \mathrm{MIC}(1.0 \mu \mathrm{g} / \mathrm{ml})$

$\rightarrow 0.5 \mathrm{MIC} \mathrm{Clr}(0.13 \mu \mathrm{g} / \mathrm{ml})+$ Golmmune Strong $(0.39 \mathrm{mg} / \mathrm{ml})$

- $1 \mathrm{MIC} \mathrm{Clr}(0.25 \mu \mathrm{g} / \mathrm{ml})+$ Golmmune Strong $(0.78 \mathrm{mg} / \mathrm{ml})$

๑- 2MIC Clr $(0.5 \mu \mathrm{g} / \mathrm{ml})+$ Golmmune Strong $(1.56 \mathrm{mg} / \mathrm{ml})$

\& $4 \mathrm{MIC} \mathrm{Clr}(1.0 \mu \mathrm{g} / \mathrm{ml})+$ Golmmune Strong $(3.12 \mathrm{mg} / \mathrm{ml})$

Figure 2. Time-kill curves show synergistic interaction between GoImmune Strong ${ }^{\circledR}$ complex and clarithromycin (Clr) against S. aureus. (A) - time-kill curves of GoImmune Strong ${ }^{\circledR}$ complex at different concentrations. (B)-Time-kill curves of combination of GoImmune Strong ${ }^{\circledR}$ complex and clarithromycin. Each time-curve experiment was performed in duplicate and diluted samples were plated in duplicate on agar plates. Error bars indicate standard deviations.

Clarithromycin did not have an effect on colony counts of $S$. aureus. GoImmune Strong ${ }^{\circledR}$ complex alone did not reduce colony counts within the first $6 \mathrm{~h}$ of incubation. However, the differences in colony counts were statistically significant compared to control $(p<0.05)$. A statistically significant reduction by $1.28 \mathrm{Log}$ was seen after $24 \mathrm{~h}$ for a $2 \mathrm{MIC}$ concentration; in the presence of $4 \mathrm{MIC}$, no viable bacteria were detected. An effect of the combination of GoImmune Strong ${ }^{\circledR}$ complex with the antibiotic was seen after $6 \mathrm{~h}-$ all tested concentrations reduced colony counts. The reduction in presence of antibiotic and GoImmune Strong ${ }^{\circledR}$ combination was statistically significant $(p<0.001)$ compared to clarithromycin alone at all concentrations. After $24 \mathrm{~h}$, a $1.96 \log _{10}$ reduction was observed in the presence of $1 \mathrm{MIC}$ and 2 MIC concentrations; no viable bacteria were detected in the presence of a $4 \mathrm{MIC}$ concentration of the combination.

\section{Discussion}

Natural compounds from various sources, including plants and bee products, have been characterized for various biological activities. Among them, antimicrobial activity is of particular interest in light of increasing antibiotic resistance and emerging new strains of pathogens. A promising approach is to use plant extracts or isolated compounds as boosters of antibiotic activity and tools for the restoration of susceptibility in resistant microorganisms $[80,81]$. The combination of various natural products and in-depth char- 
acterization of their biological activities is an important precondition for the successful repurposing of existing products for the prevention and treatment of infections.

In this study, a combination of commercially available plant and propolis extracts that is currently used as a food supplement, GoImmune Strong ${ }^{\circledR}$, was tested for its antibacterial characteristics. GoImmune Strong ${ }^{\circledR}$ was developed based on available data on antioxidative and immunity boosting properties of chosen plant extracts. Following market launch, observations and feedbacks from customers indicated on the potential positive effects of the product in respiratory infections. This urged to us to assess GoImmune Strong ${ }^{\circledR}$ for its activity against selected respiratory pathogens and interaction with antibiotics to estimate its potential to be repurposed as a prophylactic and antimicrobial therapy-enhancing product. Two of the components of the GoImmune Strong ${ }^{\circledR}$ complex, O.europae leaf extract and Brazilian green propolis extract, have been thoroughly studied for their biological activities. The third component, T. avellanedae, despite the wide traditional usage, is less characterized. To the best of our knowledge, there are no previous studies on biological activities of the combination of extracts of these three natural products.

Results showed that the complex of the extracts inhibits the growth of all tested microorganisms, with the most pronounced effect against $S$. aureus. This is in line with the published studies about individual components of the complex. Anti-staphylococcal activity is characteristic for all three extracts. Compared to some published studies, the MIC value of complex exceeds that of individual extracts, e.g., $2.68 \mathrm{mg} / \mathrm{mL}$ of olive leaf extract reported by Pereira et al. and $2.5 \mathrm{mg} / \mathrm{mL}$ by Karygianni et al., compared to $0.78 \mathrm{mg} / \mathrm{mL}$ in our study [21,23]; some other authors reported higher activity, e.g., a MIC value of $15.6 \mu \mathrm{g} / \mathrm{mL}$ of olive extract against MRSA [82], or similar results, such as MIC values for propolis in the range of $0.39-0.78 \mathrm{mg} / \mathrm{mL}$ [41]. In general, data about propolis and olive leaf extract activity against $S$. aureus vary widely among studies due to different collection sites and extraction methods [22,28,29,44,48,83]. The same applies to total phenolic content, where variations of concentrations mainly arise from different extraction methodologies.

$H$. influenzae and K. pneumoniae were less susceptible than Gram-positive S. aureus, a result that is similar to the effects of polyphenol-rich propolis extracts reported in the literature $[44,84]$. Tabebuia spp. are also reported to have lower or no activity against Gram-negative bacteria $[54,55,57]$. It is hypothesized that the high activity of the complex against $S$. aureus is due to the combined anti-staphylococcal effect of all three extracts, but the ability to inhibit the growth of Gram-negative H. influenzae and K. pneumoniae is due to the broader antimicrobial activity of olive leaf extract.

Natural extracts are complex mixtures of various compounds that possess multiple mechanisms of action. Synergistic activities between different extract components are possible. Some compounds identified in the components of GoImmune Strong ${ }^{\circledR}$ are known for their activity against S. aureus, K. pneumoniae and H. influenzae. Hydroxytyrosol and oleuropein has been shown to be effective against all three bacteria [58,85-87]. Other broad spectrum compounds of GoImmune Strong ${ }^{\circledR}$ that are effective against both Grampositive and Gram-negative bacteria are cinnamic acid and its derivatives, verbascoside and isoverboscaside [88,89]. 4-hydroxybenzoic acid was detected in T.avellanedae bark extract and olive leaf extract and is well known for its activity against Gram-positive bacteria [90]. The modes of action of phenolic compounds are not yet fully elucidated. The variations in the activity against Gram-negative and Gram-positive bacteria might be explained by the differences in the cell surface. It has been proposed that the outer membrane of Gramnegative bacteria blocks penetration of antimicrobial compounds, making bacteria less susceptible [68]. Other studies point out that there is no clear correlation between Gram staining and susceptibility to phenolic compounds. Susceptibility varies between bacterial species or even strains and is dependent on the physico-chemical characteristics of the compounds [91].

A valuable finding of our study is the synergistic activity of the GoImmune Strong ${ }^{\circledR}$ complex with antibiotics used to treat respiratory infections. Synergy against $H$. influenzae was detected for all tested antibiotics. Synergy with azithromycin and clarithromycin 
against S. aureus was observed. Against K. pneumoniae, the complex was synergistic only with clarithromycin. Synergy could be explained by the activity of individual extracts of the complex. In the literature, the synergy of propolis extracts with antibiotics acting on the bacterial cell wall and protein synthesis has been reported [41,43]. Olive leaf extracts have synergistic activities with beta-lactams [28]. The specific mechanisms of synergy and role of individual compounds is yet to be elucidated. It is hypothesized that synergistic activity arises from different targets and mechanisms of action of the components of the complex and antibiotics.

The results of our study show the potential of the GoImmune Strong ${ }^{\circledR}$ complex to be used as an antibiotic booster, allowing for the enhancement of the efficacy of antibiotic therapy as well as a reduction in the administered concentrations of antibiotics for respiratory infections. At the same time, it is clear that further studies are needed to characterize the mechanisms of synergy and prove the antimicrobial activity in vivo. Tests in antibiotic-resistant microbial strains would be beneficial to further prove the applicability of the extract complex in the treatment of infections.

\section{Materials and Methods}

\subsection{Extracts and Their Preparation for the Tests}

Green propolis extract (BNatural, Corbetta, Italy), standardized to contain 5\% total phenolic acids; Tabebuia avellanedae bark extract (EPO Instituto Farmochimico Fitoterapico, Milan, Italy), standardized to contain 3\% w/w lapachol; and Olea europaea leaf extract (Gonmisol, Barcelona, Spain) standardized to contain $20 \% w / w$ oleuropein were combined in a ratio of $1: 2.5: 2$, respectively. Ratio of the extracts was chosen to be identical to that of the commercial product GoImmune Strong ${ }^{\circledR}$.

Prior to antimicrobial activity tests, complex was dissolved in dimethyl sulfoxide (Sigma-Aldrich, St. Louis, MO, USA) at concentration $200 \mathrm{mg} / \mathrm{mL}$.

For total phenolic content analysis all plant extracts were dissolved in dimethyl sulfoxide (Sigma-Aldrich, St. Louis, MO, USA) at concentration $50 \mathrm{mg} / \mathrm{mL}$; green propolis extract was dissolved in dimethyl sulfoxide at concentration $25 \mathrm{mg} / \mathrm{mL}$.

\subsection{Total Phenolic Content}

Total phenolic content (TPC) was determined using Folin-Ciocalteu assay adjusted for microplates with gallic acid as the standard. Briefly, dilutions of extracts $(25 \mu \mathrm{L})$ in $75 \mu \mathrm{L}$ water were incubated with $25 \mu \mathrm{L} 1 \mathrm{~N}$ Folin-Ciocalteu reagent (Sigma-Aldrich, St. Louis, $\mathrm{MO}, \mathrm{USA}$ ) for $6 \mathrm{~min}$ at room temperature (22-24oC). A total of $100 \mu \mathrm{L} \mathrm{7 \%}$ sodium carbonate was added to the reaction. The absorbance was measured at $760 \mathrm{~nm}$ using a microplate reader (TECAN Infinite 200PRO) after incubation for $90 \mathrm{~min}$ at room temperature in the dark. All samples were analyzed in duplicates, with three technical replicates. Results were expressed as mg of gallic acid equivalent (GAE) per g of dry weight (DW).

\subsection{Chromatographic Analysis}

\subsubsection{Preparation of Samples and Standards}

The method of Ghomari et al., 2019 [58], was adapted for sample preparation of green propolis, Tabebuia avellanedae bark, and Olea europaea leaf extract. A total of $0.2 \mathrm{~g}$ of dried plant extracts was mixed with $2 \mathrm{~mL}$ of $80 \%(v / v)$ ethanol for $4 \mathrm{~h}$. Samples were filtered through a nylon membrane filter (pore size $0.45 \mu \mathrm{m}$ ) and were injected into the HPLC system.

External standard calibration was used for quantitative determination of phenolic compounds and oleuropein. Analytical standards of oleuropein, chlorogenic acid, caffeic acid, p-coumaric acid, ferulic acid, naringenin, rutin, vanillic acid, cinnamic acid, 4-hydroxy benzoic acid, and protocatechuic acid were purchased from Sigma-Aldrich. Stock solutions of standards at a concentration of $1000 \mathrm{mg} / \mathrm{L}$ were prepared in mobile phase. Working solutions were prepared ranging from 0.5 to $25 \mathrm{mg} / \mathrm{L}$ by diluting the stock solutions with mobile phase. All stock and working solutions were stored at $4{ }^{\circ} \mathrm{C}$ temperature. The 
standard solution at each concentration was analyzed in triplicate. All calibration curves were constructed by plotting the average peak area against concentration.

\subsubsection{HPLC-TOF-HRMS Analysis}

The chemical composition of the ethanolic extracts was determined using an Agilent 1290 Infinity series system (Agilent Technologies, Waldbronn, Germany) coupled to an Agilent 6230 TOF LC/MS (Agilent Technologies, Waldbronn, Germany) with electrospray ionization (ESI). Chromatographic separation of phenolic compounds and iridoids was performed at $30{ }^{\circ} \mathrm{C}$ using an Xterra MS C18, $2.1 \times 150 \mathrm{~mm}, 3.5 \mu \mathrm{m}$ column. The mobile phase consisted of aqueous $0.1 \%$ formic acid (A) and acetonitrile (B). The flow rate was

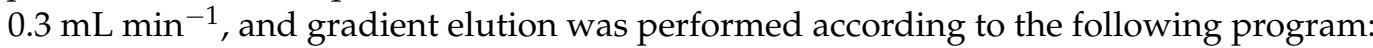
$0 \mathrm{~min}, 2 \% \mathrm{~B} ; 5.0 \mathrm{~min}, 2 \% \mathrm{~B} ; 20.0 \mathrm{~min}, 95 \% \mathrm{~B} ; 25.0 \mathrm{~min}, 95 \% \mathrm{~B} ; 26.0 \mathrm{~min}, 2 \% \mathrm{~B} ; 30.0 \mathrm{~min}, 2 \%$ B. The injection volume was $20 \mu \mathrm{L}$.

The mass spectrometry operating conditions were as follows in negative ionization mode: gas temperature $320^{\circ} \mathrm{C}$, gas flow rate $12 \mathrm{~L} / \mathrm{min}$, nebulizer pressure 40 psi, sheath gas temperature $320^{\circ} \mathrm{C}$, sheath gas flow $12 \mathrm{~L} / \mathrm{min}$, capillary voltage $4000 \mathrm{~V}$, and applied fragmentor $130 \mathrm{~V}$. The full scan mass range was set to 50-1500 m/z. Internal reference masses $112.98559 \mathrm{~m} / \mathrm{z}$ and $1033.98811 \mathrm{~m} / \mathrm{z}$ (G1969-85001 ES-TOF Reference Mass Solution Kit, Agilent Technologies \& Supelco) were used. Spectral UV data from all peaks were accumulated in the range $200-600 \mathrm{~nm}$, and chromatograms were monitored at 260, 280, and $520 \mathrm{~nm}$. Spectrum extraction and peak detection were performed with MassHunter 7.00 Software (Agilent). Peak identification was determined by $m / z$ values and standards.

\subsection{Determination of MIC and MBC}

Mueller-Hinton broth (Biolife, Milan, Italy) was used for susceptibility testing by twofold serial broth microdilution of Staphylococcus aureus MSCL 334 and Klebsiella pneumoniae MSCL 535 in aerobic conditions. Mueller-Hinton broth supplemented with yeast extract $5 \mathrm{mg} / \mathrm{mL}$, hemin $15 \mu \mathrm{g} / \mathrm{mL}$, and NAD $15 \mu \mathrm{g} / \mathrm{mL}$ was used for testing of Haemophilus influenzae MSCL 1619 in anaerobic conditions (GasPak Anaerobe Pouch, BD, USA).

A total of $10 \mathrm{mg} / \mathrm{mL}$ stock solution of antibiotics was prepared. Amoxiclav (Sandoz, Kundl, Austria; amoxicillin 875 mg, acid clavulanic $125 \mathrm{mg}$ ) was dissolved in sterile water. Azithromycin dihydrate (Sigma-Aldrich, St. Louis, MO, USA) was dissolved in ethanol and further diluted in water. Clarithromycin (Sigma-Aldrich, St. Louis, MO, USA) was dissolved in dimethyl sulfoxide (Sigma-Aldrich, St. Louis, MO, USA) and further diluted in water. Stock suspension of extract complex was prepared in dimethyl sulfoxide at concentration $200 \mathrm{mg} / \mathrm{mL}$. Solutions of antibiotics and suspension of extract complex with different concentrations were freshly prepared on the day of the experiment.

The inoculum of bacteria was prepared in sterile water with density of $0.08-0.10$ at $\mathrm{A}_{625}$ and diluted 100-fold in appropriate broth. Then, 96-well plates were incubated at $37^{\circ} \mathrm{C}$ for $24 \mathrm{~h}$. The MIC was determined as the lowest concentration of studied material, which showed no visible growth. From wells where growth was not detected, $4 \mu \mathrm{L}$ of media was seeded on appropriate solidified media for MBC determination.

\subsection{Determination of Fractional Inhibitory Concentrations (FIC) and FIC Index (FICI)}

The combined effect of extract combination and antibiotics (FIC) was evaluated by modified microdilution chequerboard method (Fratini et al., 2017). Assay was performed on 96-well plate using previously determined MIC values. Seven concentrations of the extract combination were prepared (4 MIC, 2 MIC, MIC, 1/2 MIC, 1/4 MIC, 1/8 MIC, and $1 / 16 \mathrm{MIC}$ ). Dilutions of the extract combination were added on the $x$-axis across the chequerboard plate, while dilutions of antibiotic were dispensed on the $y$-axis in order to obtain six final concentrations (4 MIC, 2 MIC, MIC, 1/2 MIC, 1/4 MIC, and 1/8 MIC). The inoculum of bacteria was prepared in sterile water with density of $0.08-0.10$ at $\mathrm{A}_{625}$ and diluted 100-fold in appropriate broth. Microplates were incubated at $37^{\circ} \mathrm{C}$ for $24 \mathrm{~h}$. FIC determinations were performed in triplicate. 
FICI values were calculated using the following formula:

$$
\mathrm{FICI}=\mathrm{FIC}_{\text {antibiotic }}+\mathrm{FIC}_{\text {Complex }}
$$

where

$$
\mathrm{FIC}_{\text {antibiotic }}=\mathrm{MIC}_{\text {antibiotic }} \text { in combination } / \mathrm{MIC}_{\text {antibiotic }} \text { alone }
$$

and

$$
\mathrm{FIC}_{\text {Complex }}=\mathrm{MIC}_{\text {Complex }} \text { in combination } / \mathrm{MIC}_{\text {Complex }} \text { alone. }
$$

Three different FICI interpretation methods were used to evaluate results. According to Fratini et al., 2017 [79], a synergistic effect $\left(\mathrm{Syn}_{\mathrm{A}}\right)$ is detected when FICI value $<1$, a commutative effect $\left(\mathrm{Com}_{\mathrm{A}}\right)$ when FICI value $=1$, an indifferent effect $\left(\operatorname{Ind}_{\mathrm{A}}\right)$ when $1<\mathrm{FICI}$ value $\leq 2$, and an antagonistic effect $\left(\right.$ Ant $\left._{\mathrm{A}}\right)$ when $\mathrm{FICI}$ value $>2$.

According to Odds 2003 [78], a synergistic effect (Syn $)$ is observed when FICI value $\leq 0.5$; an indifferent effect $\left(\operatorname{Ind}_{\mathrm{O}}\right)$ when $0.5<$ FICI value $\leq 4$, and an antagonistic effect $\left(\right.$ Ant $\left._{\mathrm{O}}\right)$ when FICI value $>4$.

According to EUCAST 2000 [77], a synergistic effect $\left(\right.$ Syn $\left._{\mathrm{E}}\right)$ is observed when FICI value $\leq 0.5$, an additive effect $\left(\mathrm{Add}_{\mathrm{E}}\right)$ when $0.5<$ FICI value $\leq 1$, an indifferent effect $\left(\right.$ Ind $\left._{\mathrm{E}}\right)$ when $1<$ FICI value $<2$, and an antagonistic effect $\left(\right.$ Ant $\left._{E}\right)$ when FICI value $\geq 2$.

\subsection{Time-Kill Assay}

The concentrations of half the MIC, equal to MIC, twice the MIC, and four times the MIC of the extract complex and antibiotics estimated in MIC assays were prepared. A bacterial inoculum with a final concentration of $10^{6} \mathrm{CFU} / \mathrm{mL}$ was added and incubated at $37^{\circ} \mathrm{C}$ in an appropriate broth. A bacterial inoculum without added substances was used as a control. Aliquots of $0.1 \mathrm{~mL}$ in each tube were taken at time intervals of $0,3,6$, and $24 \mathrm{~h}$. Ten-fold serial dilutions were prepared and inoculated on appropriate solidified media and incubated at $37^{\circ} \mathrm{C}$ for $24-48 \mathrm{~h}$. The number of colony-forming units (CFU) was determined. A graph of the $\log _{10} \mathrm{CFU} / \mathrm{mL}$ was plotted against time. Each time-curve experiment was performed in duplicate and diluted samples were plated in duplicate on agar plates. Error bars indicate standard deviations.

\subsection{Data Analysis}

Data were analyzed and graphs generated using GraphPad Prism 5.0 software (San Diego, CA, USA). Two-way ANOVA followed by multiple comparison test was used to test for differences between antibiotic combinations with GoImmune Strong ${ }^{\circledR}$ and single concentrations over time. Differences were considered statistically significant if $p \leq 0.05$.

\section{Conclusions}

In the era of antibiotic resistance, phenolic substances have become a subject of particular interest in prophylaxis and handling of bacterial infections. The testing of already available complexes of polyphenol-rich extracts revealed the antimicrobial activity against respiratory pathogens $S$. aureus, K. pneumoniae, and $H$. influenzae. The synergistic activity with beta-lactam and protein-synthesis-inhibiting antibiotics against both Gram-negative and Gram-positive bacteria points to the high potential of the complex to be applied in prophylaxis and treatment of respiratory infections. The mechanism of action and role of individual compounds in provision of the activity needs to be further investigated. The results of the study are an essential precondition for successful further repurposing of the polyphenol complex for use against bacterial infections. To achieve this, additional in vitro tests using multidrug-resistant strains are needed, followed by in vivo efficacy studies.

Author Contributions: Conceptualization, V.N., L.G. and E.G.; methodology, Z.P., V.V. and A.R.-S.; formal analysis, V.N.; investigation, Z.P., V.V., A.R.-S. and M.B.; writing-original draft preparation, A.R.-S.; project administration, M.B. All authors have read and agreed to the published version of the manuscript. 
Funding: This research was funded by Silvanols Ltd.

Institutional Review Board Statement: Not applicable.

Informed Consent Statement: Not applicable.

Data Availability Statement: Not applicable.

Conflicts of Interest: The authors declare no conflict of interest.

\section{References}

1. Manohar, P.; Loh, B.; Nachimuthu, R.; Hua, X.; Welburn, S.C.; Leptihn, S. Secondary Bacterial Infections in Patients with Viral Pneumonia. Front. Med. 2020, 7, 420. [CrossRef] [PubMed]

2. Santella, B.; Serretiello, E.; de Filippis, A.; Veronica, F.; Iervolino, D.; Dell'annunziata, F.; Manente, R.; Valitutti, F.; Santoro, E.; Pagliano, P.; et al. Lower Respiratory Tract Pathogens and Their Antimicrobial Susceptibility Pattern: A 5-Year Study. Antibiotics 2021, 10, 851. [CrossRef] [PubMed]

3. Duan, N.; Du, J.; Huang, C.; Li, H. Microbial Distribution and Antibiotic Susceptibility of Lower Respiratory Tract Infections Patients from Pediatric Ward, Adult Respiratory Ward, and Respiratory Intensive Care Unit. Front. Microbiol. 2020, 11, 1480. [CrossRef] [PubMed]

4. Bakaletz, L.O. Viral-Bacterial Co-Infections in the Respiratory Tract. Curr. Opin. Microbiol. 2017, 35, 30-35. [CrossRef] [PubMed]

5. Smith, R.; Coast, J. The True Cost of Antimicrobial Resistance. BMJ 2013, 346, f1493. [CrossRef] [PubMed]

6. Barker, B.L.; Brightling, C. Pharmacological Treatment of Bacterial Infections of the Respiratory Tract. Anaesth. Intensive Care Med. 2015, 16, 79-82. [CrossRef]

7. Prestinaci, F.; Pezzotti, P.; Pantosti, A. Antimicrobial Resistance: A Global Multifaceted Phenomenon. Pathog. Glob. Health 2015, 109, 309-318. [CrossRef]

8. Vaou, N.; Stavropoulou, E.; Voidarou, C.; Tsigalou, C.; Bezirtzoglou, E. Towards Advances in Medicinal Plant Antimicrobial Activity: A Review Study on Challenges and Future Perspectives. Microorganisms 2021, 9, 2041. [CrossRef]

9. Keman, D.; Soyer, F. Antibiotic-Resistant Staphylococcus Aureus Does Not Develop Resistance to Vanillic Acid and 2-Hydroxycinnamic Acid after Continuous Exposure In Vitro. ACS Omega 2019, 4, 15393-15400. [CrossRef]

10. Ofosu, F.K.; Daliri, E.B.M.; Elahi, F.; Chelliah, R.; Lee, B.H.; Oh, D.H. New Insights on the Use of Polyphenols as Natural Preservatives and Their Emerging Safety Concerns. Front. Sustain. Food Syst. 2020, 4, 525810. [CrossRef]

11. Clewell, A.E.; Béres, E.; Vértesi, A.; Glávits, R.; Hirka, G.; Endres, J.R.; Murbach, T.S.; Szakonyiné, I.P. A Comprehensive Toxicological Safety Assessment of an Extract of Olea europaea L. Leaves (Bonolive ${ }^{\mathrm{TM}}$ ). Int. J. Toxicol. 2016, 35, 208-221. [CrossRef] [PubMed]

12. Savoia, D. Plant-Derived Antimicrobial Compounds: Alternatives to Antibiotics. Future Microbiol. 2012, 7, 979-990. [CrossRef]

13. Patra, A.K. An Overview of Antimicrobial Properties of Different Classes of Phytochemicals. In Dietary Phytochemicals and Microbes; Springer: Dordrecht, The Netherlands, 2012; pp. 1-32, ISBN 978-94-007-3926-0.

14. Sanhueza, L.; Melo, R.; Montero, R.; Maisey, K.; Mendoza, L.; Wilkens, M. Synergistic Interactions between Phenolic Compounds Identified in Grape Pomace Extract with Antibiotics of Different Classes against Staphylococcus Aureus and Escherichia Coli. PLoS ONE 2017, 12, e0172273. [CrossRef] [PubMed]

15. Pancu, D.F.; Scurtu, A.; Macasoi, I.G.; Marti, D.; Mioc, M.; Soica, C.; Coricovac, D.; Horhat, D.; Poenaru, M.; Dehelean, C. Antibiotics: Conventional Therapy and Natural Compounds with Antibacterial Activity-a Pharmaco-Toxicological Screening. Antibiotics 2021, 10, 401. [CrossRef] [PubMed]

16. Atanasov, A.G.; Waltenberger, B.; Pferschy-Wenzig, E.M.; Linder, T.; Wawrosch, C.; Uhrin, P.; Temml, V.; Wang, L.; Schwaiger, S.; Heiss, E.H.; et al. Discovery and Resupply of Pharmacologically Active Plant-Derived Natural Products: A Review. Biotechnol. Adv. 2015, 33, 1582-1614. [CrossRef] [PubMed]

17. Ruddaraju, L.K.; Pammi, S.V.N.; sankar Guntuku, G.; Padavala, V.S.; Kolapalli, V.R.M. A Review on Anti-Bacterials to Combat Resistance: From Ancient Era of Plants and Metals to Present and Future Perspectives of Green Nano Technological Combinations. Asian J. Pharm. Sci. 2020, 15, 42-59. [CrossRef]

18. Coppo, E.; Marchese, A. Antibacterial Activity of Polyphenols. Curr. Pharm. Biotechnol. 2014, 15, 380-390. [CrossRef]

19. Martin, D.; Konrad, M.; Adarkwah, C.C.; Kostev, K. Reduced Antibiotic Use after Initial Treatment of Acute Respiratory Infections with Phytopharmaceuticals- a Retrospective Cohort Study. Postgrad. Med. 2020, 132, 412-418. [CrossRef]

20. Thielmann, J.; Kohnen, S.; Hauser, C. Antimicrobial Activity of Olea europaea Linné Extracts and Their Applicability as Natural Food Preservative Agents. Int. J. Food Microbiol. 2017, 251, 48-66. [CrossRef]

21. Pereira, A.P.; Ferreira, I.C.F.R.; Marcelino, F.; Valentão, P.; Andrade, P.B.; Seabra, R.; Estevinho, L.; Bento, A.; Pereira, J.A. Phenolic Compounds and Antimicrobial Activity of Olive (Olea europaea L. Cv. Cobrançosa) Leaves. Molecules 2007, 12, 1153-1162. [CrossRef]

22. Sudjana, A.N.; D’Orazio, C.; Ryan, V.; Rasool, N.; Ng, J.; Islam, N.; Riley, T.V.; Hammer, K.A. Antimicrobial Activity of Commercial Olea europaea (Olive) Leaf Extract. Int. J. Antimicrob. Agents 2009, 33, 461-463. [CrossRef] [PubMed] 
23. Karygianni, L.; Cecere, M.; Skaltsounis, A.L.; Argyropoulou, A.; Hellwig, E.; Aligiannis, N.; Wittmer, A.; Al-Ahmad, A. HighLevel Antimicrobial Efficacy of Representative Mediterranean Natural Plant Extracts against Oral Microorganisms. BioMed Res. Int. 2014, 2014, 1-8. [CrossRef] [PubMed]

24. Keskın, D.; Ceyhan, N.; Uğur, A.; Dbeys, A.D. Antimicrobial Activity and Chemical Constitutions of West Anatolian Olive (Olea europaea L.) Leaves. Agric. Environ. 2012, 10, 99-102.

25. Lee, O.H.; Lee, B.Y. Antioxidant and Antimicrobial Activities of Individual and Combined Phenolics in Olea europaea Leaf Extract. Bioresour. Technol. 2010, 101, 3751-3754. [CrossRef]

26. Hashmi, M.A.; Khan, A.; Hanif, M.; Farooq, U.; Perveen, S. Traditional Uses, Phytochemistry, and Pharmacology of Olea europaea (Olive). Evid.-Based Complementary Altern. Med. 2015, 2015, 541591. [CrossRef]

27. Borjan, D.; Leitgeb, M.; Knez, Ž.; Hrnčič, M.K. Microbiological and Antioxidant Activity of Phenolic Compounds in Olive Leaf Extract. Molecules 2020, 25, 5946. [CrossRef]

28. Lim, A.; Subhan, N.; Jazayeri, J.A.; John, G.; Vanniasinkam, T.; Obied, H.K. Plant Phenols as Antibiotic Boosters: In Vitro Interaction of Olive Leaf Phenols with Ampicillin. Phytother. Res. 2016, 30, 503-509. [CrossRef]

29. Silici, S.; Kutluca, S. Chemical Composition and Antibacterial Activity of Propolis Collected by Three Different Races of Honeybees in the Same Region. J. Ethnopharmacol. 2005, 99, 69-73. [CrossRef]

30. Ristivojević, P.; Dimkić, I.; Trifković, J.; Berić, T.; Vovk, I.; Milojkovič-Opsenica, D.; Stanković, S. Antimicrobial Activity of Serbian Propolis Evaluated by Means of MIC, HPTLC, Bioautography and Chemometrics. PLoS ONE 2016, 11, e0157097. [CrossRef]

31. Torres, A.R.; Sandjo, L.P.; Friedemann, M.T.; Tomazzoli, M.M.; Maraschin, M.; Mello, C.F.; Santos, A.R.S. Chemical Characterization, Antioxidant and Antimicrobial Activity of Propolis Obtained from Melipona Quadrifasciata Quadrifasciata and Tetragonisca Angustula Stingless Bees. Braz. J. Med. Biol. Res. 2018, 51, e7118. [CrossRef]

32. Popova, M.; Trusheva, B.; Bankova, V. Propolis of Stingless Bees: A Phytochemist's Guide through the Jungle of Tropical Biodiversity. Phytomedicine 2021, 86, 153098. [CrossRef] [PubMed]

33. Popova, M.; Giannopoulou, E.; Skalicka-Wózniak, K.; Graikou, K.; Widelski, J.; Bankova, V.; Kalofonos, H.; Sivolapenko, G.; Gaweł-Bęben, K.; Antosiewicz, B.; et al. Characterization and Biological Evaluation of Propolis from Poland. Molecules 2017, 22, 1159. [CrossRef] [PubMed]

34. Przybyłek, I.; Karpiński, T.M. Antibacterial Properties of Propolis. Molecules 2019, 24, 2047. [CrossRef] [PubMed]

35. Mavri, A.; Abramovič, H.; Polak, T.; Bertoncelj, J.; Jamnik, P.; Možina, S.S.; Jeršek, B. Chemical Properties and Antioxidant and Antimicrobial Activities of Slovenian Propolis. Chem. Biodivers. 2012, 9, 1545-1558. [CrossRef] [PubMed]

36. Wang, X.; Sankarapandian, K.; Cheng, Y.; Woo, S.O.; Kwon, H.W.; Perumalsamy, H.; Ahn, Y.J. Relationship between Total Phenolic Contents and Biological Properties of Propolis from 20 Different Regions in South Korea. BMC Complementary Altern. Med. 2016, 16, 65. [CrossRef] [PubMed]

37. Nainu, F.; Masyita, A.; Bahar, M.A.; Raihan, M.; Prova, S.R.; Mitra, S.; Emran, T.B.; Simal-Gandara, J. Pharmaceutical Prospects of Bee Products: Special Focus on Anticancer, Antibacterial, Antiviral, and Antiparasitic Properties. Antibiotics 2021, 10, 822. [CrossRef]

38. Campos, J.F.; das Santos, U.P.; da Rocha, P.D.S.; Damião, M.J.; Balestieri, J.B.P.; Cardoso, C.A.L.; Paredes-Gamero, E.J.; Estevinho, L.M.; de Picoli Souza, K.; dos Santos, E.L. Antimicrobial, Antioxidant, Anti-Inflammatory, and Cytotoxic Activities of Propolis from the Stingless Bee Tetragonisca Fiebrigi (Jataí). Evid.-Based Complementary Altern. Med. 2015, 2015, 1-11. [CrossRef]

39. Seidel, V.; Peyfoon, E.; Watson, D.G.; Fearnley, J. Comparative Study of the Antibacterial Activity of Propolis from Different Geographical and Climatic Zones. Phytother. Res 2008, 22, 1256-1263. [CrossRef]

40. Berretta, A.A.; Nascimento, A.P.; Bueno, P.C.P.; Leite Vaz, M.M.d.O.L.; Marchetti, J.M. Propolis Standardized Extract (EPP-AF $\left.{ }^{\circledR}\right)$, an Innovative Chemically and Biologically Reproducible Pharmaceutical Compound for Treating Wounds. Int. J. Biol. Sci. 2012, 8 , 512-521. [CrossRef]

41. Wojtyczka, R.D.; Dziedzic, A.; Idzik, D.; Kepa, M.; Kubina, R.; Kabała-Dzik, A.; Smoleń-Dzirba, J.; Stojko, J.; Sajewicz, M.; Wasik, T.J. Susceptibility of Staphylococcus Aureus Clinical Isolates to Propolis Extract Alone or in Combination with Antimicrobial Drugs. Molecules 2013, 18, 9623-9640. [CrossRef]

42. Orsi, R.O.; Fernandes, A.; Bankova, V.; Sforcin, J.M. Antibacterial Effects of Brazilian and Bulgarian Propolis and Synergistic Effects with Antibiotics Acting on the Bacterial DNA and Folic Acid. Nat. Prod. Res. 2012, 26, 344-349. [CrossRef] [PubMed]

43. Orsi, R.O.; Fernandes, A.; Bankova, V.; Sforcin, J.M. The Effects of Brazilian and Bulgarian Propolis in Vitro against Salmonella Typhi and Their Synergism with Antibiotics Acting on the Ribosome. Nat. Prod. Res. 2012, 26, 430-437. [CrossRef] [PubMed]

44. Grecka, K.; Kuś, P.M.; Okińczyc, P.; Worobo, R.W.; Walkusz, J.; Szweda, P. The Anti-Staphylococcal Potential of Ethanolic Polish Propolis Extracts. Molecules 2019, 24, 1732. [CrossRef] [PubMed]

45. Takaisi-Kikuni, N.B.; Schilcher, H. Electron Microscopic and Microcalorimetric Investigations of the Possible Mechanism of the Antibacterial Action of a Defined Propolis Provenance. Planta Med. 1994, 60, 222-227. [CrossRef] [PubMed]

46. Ratajczak, M.; Kaminska, D.; Matuszewska, E.; Hołderna-Kedzia, E.; Rogacki, J.; Matysiak, J. Promising Antimicrobial Properties of Bioactive Compounds from Different Honeybee Products. Molecules 2021, 26, 4007. [CrossRef]

47. Mirzoeva, O.K.; Grishanin, R.N.; Calder, P.C. Microbiological Research Antimicrobial Action of Propolis and Some of Its Components: The Effects on Growth, Membrane Potential and Motility of Bacteria. Microbiol. Res. 1997, 152, 239-246. [CrossRef]

48. AL-Ani, I.; Zimmermann, S.; Reichling, J.; Wink, M. Antimicrobial Activities of European Propolis Collected from Various Geographic Origins Alone and in Combination with Antibiotics. Medicines 2018, 5, 2. [CrossRef] 
49. Stepanović, S.; Antić, N.; Dakić, I.; Svabić-Vlahović, M. In Vitro Antimicrobial Activity of Propolis and Synergism between Propolis and Antimicrobial Drugs. Microbiol. Res. 2003, 158, 353-357. [CrossRef]

50. Salatino, A.; Salatino, M.L.F.; Negri, G. How Diverse Is the Chemistry and Plant Origin of Brazilian Propolis? Apidologie 2021, 52, 1075-1097. [CrossRef]

51. Bittencourt, M.L.F.; Ribeiro, P.R.; Franco, R.L.P.; Hilhorst, H.W.M.; de Castro, R.D.; Fernandez, L.G. Metabolite Profiling, Antioxidant and Antibacterial Activities of Brazilian Propolis: Use of Correlation and Multivariate Analyses to Identify Potential Bioactive Compounds. Food Res. Int. 2015, 76, 449-457. [CrossRef]

52. Dantas Silva, R.P.; Machado, B.A.S.; Barreto, G.d.A.; Costa, S.S.; Andrade, L.N.; Amaral, R.G.; Carvalho, A.A.; Padilha, F.F.; Barbosa, J.D.V.; Umsza-Guez, M.A. Antioxidant, Antimicrobial, Antiparasitic, and Cytotoxic Properties of Various Brazilian Propolis Extracts. PLoS ONE 2017, 12, e0172585. [CrossRef] [PubMed]

53. Nader, R.A.; Mackieh, R.; Wehbe, R.; El Obeid, D.; Sabatier, J.M.; Fajloun, Z. Beehive Products as Antibacterial Agents: A Review. Antibiotics 2021, 10, 717. [CrossRef] [PubMed]

54. El-Hawary, S.S.; Taher, M.A.; Amin, E.; AbouZid, S.F.; Mohammed, R. Genus Tabebuia: A Comprehensive Review Journey from Past Achievements to Future Perspectives. Arab. J. Chem. 2021, 14, 103046. [CrossRef]

55. Jiménez-González, F.J.; Veloza, L.A.; Sepúlveda-Arias, J.C. Anti-Infectious Activity in Plants of the Genus Tabebuia. Univ. Sci. 2013, 18, 257-267. [CrossRef]

56. Pereira, E.M.; de Barros Machado, T.; Ramos Leal, I.C.; Jesus, D.M.; de Almeida Damaso, C.R.; Pinto, A.V.; Giambiagi-deMarval, M.; Kuster, R.M.; Netto dos Santos, K.R. Tabebuia Avellanedae Naphtoquinones: Activity against Methicillin-Resistant Staphylococcal Strains, Cytotoxic Activity and in Vivo Dermal Irritability Analysis. Ann. Clin. Microbiol. Antimicrob. $2006,5,5$. [CrossRef]

57. Hamed, A.N.E.; Mahmoud, B.K.; Samy, M.N.; Kamel, M.S. An Extensive Review on Genus "Tabebuia", Family Bignoniaceae: Phytochemistry and Biological Activities (1967 to 2018). J. Herb. Med. 2020, 24, 100410. [CrossRef]

58. Ghomari, O.; Sounni, F.; Massaoudi, Y.; Ghanam, J.; Drissi Kaitouni, L.B.; Merzouki, M.; Benlemlih, M. Phenolic Profile (HPLC-UV) of Olive Leaves According to Extraction Procedure and Assessment of Antibacterial Activity. Biotechnol. Rep. 2019,23 , e00347. [CrossRef]

59. Jimenez, P.; Masson, L.; Barriga, A.; Chávez, J.; Robert, P. Oxidative Stability of Oils Containing Olive Leaf Extracts Obtained by Pressure, Supercritical and Solvent-Extraction. Eur. J. Lipid Sci. Technol. 2011, 113, 497-505. [CrossRef]

60. Poudyal, H.; Campbell, F.; Brown, L. Olive Leaf Extract Attenuates Cardiac, Hepatic, and Metabolic Changes in High Carbohydrate-, High Fat-Fed Rats. J. Nutr. 2010, 140, 946-953. [CrossRef]

61. Korukluoglu, M.; Sahan, Y.; Yigit, A.; Ozer, E.T.; GÜCer, S. Antibacterial Activity and Chemical Constitutions of Olea europaea 1. Leaf Extracts. J. Food Processing Preserv. 2010, 34, 383-396. [CrossRef]

62. Mylonaki, S.; Kiassos, E.; Makris, D.P.; Kefalas, P. Optimisation of the Extraction of Olive (Olea europaea) Leaf Phenolics Using Water/Ethanol-Based Solvent Systems and Response Surface Methodology. Anal. Bioanal. Chem. 2008, 392, 977-985. [CrossRef]

63. Lipid Maps. Available online: https://www.lipidmaps.org (accessed on 7 January 2022).

64. Andrikopoulos, N.K.; Salta, F.N.; Mylona, A.; Chiou, A.; Boskou, G. Oxidative Stability of Edible Vegetable Oils Enriched in Polyphenols with Olive Leaf Extract. Food Sci. Technol. Int. 2007, 13, 413-421. [CrossRef]

65. Dekanski, D.; Janićijević-Hudomal, S.; Tadić, V.; Marković, G.; Arsić, I.; Mitrović, D.M. Phytochemical Analysis and Gastroprotective Activity of an Olive Leaf Extract. J. Serb. Chem. Soc. 2009, 74, 367-377. [CrossRef]

66. El-Hawary, S.S.; Mohammed, R.; Tawfike, A.F.; AbouZid, S.F.; Taher, M.A.; Abdelmohsen, U.R.; Amin, E. Metabolic Profiling of Cytotoxic Metabolites from Five Tabebuia Species Supported by Molecular Correlation Analysis. Sci. Rep. 2021, 11, 8405. [CrossRef] [PubMed]

67. Nocchi, S.R.; Kato, N.N.; de Almeida, J.M.; Ferreira, A.M.T.; Toffoli-Kadri, M.C.; de Freitas Meirelles, L.E.; Damke, G.M.Z.F.; Consolaro, M.E.L.; Rigo, G.V.; Macedo, A.J.; et al. Pharmacological Properties of Specioside from the Stem Bark of Tabebuia Aurea. Rev. Bras. De Farmacogn. 2020, 30, 118-122. [CrossRef]

68. Inouye, S.; Yamaguchi, H.; Takizawa, T. Screening of the Antibacterial Effects of a Variety of Essential Oils on Respiratory Tract Pathogens, Using a Modified Dilution Assay Method. J. Infect. Chemother. 2001, 7, 251-254. [CrossRef]

69. de Abreu, M.B.; Temraz, A.; Vassallo, A.; Braca, A.; de Tommasi, N. Phenolic Glycosides from Tabebuia Argentea and Catalpa Bignonioides. Phytochem. Lett. 2014, 7, 85-88. [CrossRef]

70. Takahashi, S.; Kawakami, S.; Sugimoto, S.; Matsunami, K.; Otsuka, H. Lignan Glycosides and Phenolic Compound Glycosides from the Branches of Tabebuia Chrysotricha. Am. J. Plant Sci. 2015, 6, 676-684. [CrossRef]

71. Fujimoto, Y.; Eguchi, T.; Murasaki, C.; Ohashi, Y.; Kakinuma, K.; Takagaki, H.; Abe, M.; Inazawa, K.; Yamazaki, K.; Ikekawa, N.; et al. Studies on the Structure and Stereochemistry of Cytotoxic Furanonaphthoquinones from Tabebuia Impetiginosa: 5-and 8-Hydroxy-2-(1-Hyd Roxyethyl)Naphtho[2,3-b]f Uran-4,g-Diones. J. Chem. Soc., Perkin Trans. 1 1991, 10, 2323-2327. [CrossRef]

72. de Morais, S.K.R.; Silva, S.G.; Portela, C.N.; Nunomura, S.M.; Quignard, E.L.J.; Pohlit, A.M. Bioactive Dihydroxyfuranonaphthoquinones from the Bark of Tabebuia Incana A.H. Gentry (Bignoniaceae) and HPLC Analysis of Commercial Pau d'arco and Certified T.Incana Bark Infusions. Acta Amaz. 2007, 37, 99-102. [CrossRef]

73. Szliszka, E.; Kucharska, A.Z.; Sokół-Łętowska, A.; Mertas, A.; Czuba, Z.P.; Król, W. Chemical Composition and Anti-Inflammatory Effect of Ethanolic Extract of Brazilian Green Propolis on Activated J774A.1 Macrophages. Evid.-Based Complementary Altern. Med. 2013, 2013, 1-13. [CrossRef] [PubMed] 
74. Nakajima, Y.; Shimazawa, M.; Mishima, S.; Hara, H. Water Extract of Propolis and Its Main Constituents, Caffeoylquinic Acid Derivatives, Exert Neuroprotective Effects via Antioxidant Actions. Life Sci. 2007, 80, 370-377. [CrossRef] [PubMed]

75. de Moura, S.A.; Negri, G.; Salatino, A.; Lima, L.D.; Dourado, L.P.; Mendes, J.B.; Andrade, S.P.; Ferreira, M.A.; Cara, D.C Aqueous Extract of Brazilian Green Propolis: Primary Components, Evaluation of Inflammation and Wound Healing by Using Subcutaneous Implanted Sponges. Evid. -Based Complementary Altern. Med. 2011, 2011, 748283. [CrossRef] [PubMed]

76. Pellati, F.; Orlandini, G.; Pinetti, D.; Benvenuti, S. HPLC-DAD and HPLC-ESI-MS/MS Methods for Metabolite Profiling of Propolis Extracts. J. Pharm. Biomed. Anal. 2011, 55, 934-948. [CrossRef] [PubMed]

77. European Committee for Antimicrobial Susceptibility Testing (EUCAST) of the European Society of Clinical Microbiology and Infectious Dieases (ESCMID). EUCAS Definitive document E.Def 1.2, May 2000: Terminology Relating to Methods for the Determination of Susceptibility of Bacteria to Antimicrobial Agents European Committee ForAntimicrobial SusceptibilityTesting (EUCAST) of the European Society of Clinical Microbiology and Infectious Diseases (ESCMID). Clin. Microbiol. Infect. 2000, 6, 503-508. [CrossRef]

78. Odds, F.C. Synergy, Antagonism, and What the Chequerboard Puts between Them. J. Antimicrob. Chemother. 2003, 52, 1. [CrossRef]

79. Fratini, F.; Mancini, S.; Turchi, B.; Friscia, E.; Pistelli, L.; Giusti, G.; Cerri, D. A Novel Interpretation of the Fractional Inhibitory Concentration Index: The Case Origanum Vulgare L. and Leptospermum Scoparium J. R. et G. Forst Essential Oils against Staphylococcus Aureus Strains. Microbiol. Res. 2017, 195, 11-17. [CrossRef]

80. Borges, A.; Abreu, A.C.; Dias, C.; Saavedra, M.J.; Borges, F.; Simões, M. New Perspectives on the Use of Phytochemicals as an Emergent Strategy to Control Bacterial Infections Including Biofilms. Molecules 2016, 21, 877. [CrossRef]

81. Hemaiswarya, S.; Kruthiventi, A.K.; Doble, M. Synergism between Natural Products and Antibiotics against Infectious Diseases. Phytomedicine 2008, 15, 639-652. [CrossRef]

82. Elnahas, R.A.; Elwakil, B.H.; Elshewemi, S.S.; Olama, Z.A. Egyptian Olea europaea Leaves Bioactive Extract: Antibacterial and Wound Healing Activity in Normal and Diabetic Rats. J. Tradit. Complementary Med. 2021, 11, 427-434. [CrossRef]

83. Massaro, C.F.; Simpson, J.B.; Powell, D.; Brooks, P. Chemical Composition and Antimicrobial Activity of Honeybee (Apis Mellifera Ligustica) Propolis from Subtropical Eastern Australia. Sci. Nat. 2015, 102, 68. [CrossRef] [PubMed]

84. Nina, N.; Quispe, C.; Jiménez-Aspee, F.; Theoduloz, C.; Feresín, G.E.; Lima, B.; Leiva, E.; Schmeda-Hirschmann, G. Antibacterial Activity, Antioxidant Effect and Chemical Composition of Propolis from the Región Del Maule, Central Chile. Molecules 2015, 20, 18144-18167. [CrossRef] [PubMed]

85. Azaizeh, H.; Tafesh, A.; Najami, N.; Jadoun, J.; Halahlih, F.; Riepl, H. Synergistic Antibacterial Effects of Polyphenolic Compounds from Olive Mill Wastewater. Evid.-Based Complementary Altern. Med. 2011, 2011, 431021. [CrossRef]

86. Yuan, J.J.; Yan, H.J.; He, J.; Liu, Y.Y. Antibacterial Activities of Polyphenols from Olive Leaves against Klebsiella Pneumoniae. In Proceedings of the IOP Conference Series: Earth and Environmental Science, 6th International Symposium on Energy Science and Chemical Engineering, Harbin, China, 22-24 January 2021; IOP Publishing Ltd.: Bristol, UK, 2021; Volume 680. [CrossRef]

87. Bisignano, G.; Tomaino, A.T.; lo Cascio, R.; Crisafi, G.; Uccella, N.; Saija, A. On the In-Vitro Antimicrobial Activity of Oleuropein and Hydroxytyrosol. J. Pharm. Pharm. 1999, 51, 971-974. [CrossRef]

88. Guzman, J.D. Natural Cinnamic Acids, Synthetic Derivatives and Hybrids with Antimicrobial Activity. Molecules 2014, 19, 19292-19349. [CrossRef]

89. Kubica, P.; Szopa, A.; Kokotkiewicz, A.; Miceli, N.; Taviano, M.F.; Maugeri, A.; Cirmi, S.; Synowiec, A.; Gniewosz, M.; Elansary, H.O.; et al. Production of Verbascoside, Isoverbascoside and Phenolic Acids in Callus, Suspension, and Bioreactor Cultures of Verbena Officinalis and Biological Properties of Biomass Extracts. Molecules 2020, 25, 5609. [CrossRef]

90. Cho, J.-Y.; Moon, J.-H.; Seong, K.-Y.; Park, K.-H. Antimicrobial Activity of 4-Hydroxybenzoic Acid and Trans 4-Hydroxycinnamic Acid Isolated and Identified from Rice Hull. Biosci. Biotechnol. Biochem. 1998, 62, 2273-2276. [CrossRef]

91. Bouarab-Chibane, L.; Forquet, V.; Lantéri, P.; Clément, Y.; Léonard-Akkari, L.; Oulahal, N.; Degraeve, P.; Bordes, C. Antibacterial Properties of Polyphenols: Characterization and QSAR (Quantitative Structure-Activity Relationship) Models. Front. Microbiol. 2019, 10, 829. [CrossRef] 